\title{
MIXED FINITE ELEMENT APPROXIMATION OF PERIODIC HAMILTON-JACOBI-BELLMAN PROBLEMS WITH APPLICATION TO NUMERICAL HOMOGENIZATION*
}

\author{
DIETMAR GALLISTL ${ }^{\dagger}$, TIMO SPREKELER ${ }^{\ddagger}$, AND ENDRE SÜLI ${ }^{\ddagger}$
}

\begin{abstract}
In the first part of the paper, we propose and rigorously analyze a mixed finite element method for the approximation of the periodic strong solution to the fully nonlinear second-order Hamilton-Jacobi-Bellman equation with coefficients satisfying the Cordes condition. These problems arise as the corrector problems in the homogenization of Hamilton-Jacobi-Bellman equations. The second part of the paper focuses on the numerical homogenization of such equations, more precisely on the numerical approximation of the effective Hamiltonian. Numerical experiments demonstrate the approximation scheme for the effective Hamiltonian and the numerical solution of the homogenized problem.
\end{abstract}

Key words. Hamilton-Jacobi-Bellman equation, nondivergence-form elliptic PDE, Cordes condition, mixed finite element methods, homogenization

AMS subject classifications. 35B27, 35J60, 65N12, 65N15, 65N30

DOI. $10.1137 / 20 \mathrm{M} 1371397$

1. Introduction. In the first part of this work we consider the numerical approximation of the periodic boundary-value problem for the elliptic Hamilton-JacobiBellman (HJB) equation

$$
\sup _{\alpha \in \Lambda}\left\{L^{\alpha} u-f^{\alpha}\right\}=0 \quad \text { in } Y, \quad u \text { is } Y \text {-periodic, }
$$

where $\Lambda$ denotes a compact metric space and $Y:=(0,1)^{n} \subset \mathbb{R}^{n}$ is the unit cell in dimension $n \geq 2$. Here, $\left\{L^{\alpha}\right\}_{\alpha \in \Lambda}$ denotes the parametrized family of the linear uniformly elliptic (see (2.2)) differential operators

$$
\begin{aligned}
L^{\alpha} w & :=-A^{\alpha}: D^{2} w-b^{\alpha} \cdot \nabla w+c^{\alpha} w \\
& :=-\sum_{i, j=1}^{n} a_{i j}(\cdot, \alpha) \partial_{i j}^{2} w-\sum_{i=1}^{n} b_{i}(\cdot, \alpha) \partial_{i} w+c(\cdot, \alpha) w
\end{aligned}
$$

and $f^{\alpha}:=f(\cdot, \alpha)$ with uniformly continuous functions $a_{i j}=a_{j i}, b_{i}, c, f \in C\left(\mathbb{R}^{n} \times \Lambda\right)$ and positive zeroth-order coefficient $c>0$.

It is assumed that $A^{\alpha}, b^{\alpha}, c^{\alpha}, f^{\alpha}$ are $Y$-periodic on $\mathbb{R}^{n}$ and that the coefficients satisfy the Cordes condition, i.e., that there exist constants $\lambda>0$ and $\delta \in(0,1)$ such that

$$
\left|A^{\alpha}\right|^{2}+\frac{\left|b^{\alpha}\right|^{2}}{2 \lambda}+\frac{\left(c^{\alpha}\right)^{2}}{\lambda^{2}} \leq \frac{1}{n+\delta}\left(\operatorname{tr}\left(A^{\alpha}\right)+\frac{c^{\alpha}}{\lambda}\right)^{2}
$$

\footnotetext{
*Received by the editors October 5, 2020; accepted for publication (in revised form) March 30, 2021; published electronically June 16, 2021.

https://doi.org/10.1137/20M1371397

Funding: This work was supported by the UK Engineering and Physical Sciences Research Council [EP/L015811/1].

${ }^{\dagger}$ Friedrich-Schiller-Universität Jena, Institut für Mathematik, Ernst-Abbe-Platz 2, 07743 Jena, Germany (dietmar.gallistl@uni-jena.de).

${ }^{\ddagger}$ University of Oxford, Mathematical Institute, Woodstock Road, Oxford OX2 6GG, UK (sprekeler@maths.ox.ac.uk, suli@maths.ox.ac.uk).
} 
holds in $\mathbb{R}^{n}$ for all $\alpha \in \Lambda$. Under these assumptions, the periodic HJB problem (1.1) admits a unique strong solution $u \in H_{\text {per }}^{2}(Y)$; see section 2.1.

Problems of the form (1.1) arise naturally in the homogenization of HJB equations, which is the focus of the second part of this work. We are concerned with elliptic homogenization problems of the form

$$
\left\{\begin{aligned}
u_{\varepsilon}+F\left[x, \frac{x}{\varepsilon}, \nabla u_{\varepsilon}, D^{2} u_{\varepsilon}\right] & =0 & & \text { in } \Omega, \\
u_{\varepsilon} & =0 & & \text { on } \partial \Omega
\end{aligned}\right.
$$

with $\Omega \subset \mathbb{R}^{n}$ being a convex domain in dimension $n \geq 2$, a small parameter $\varepsilon>0$, and

$$
F\left[x, y, \nabla w, D^{2} w\right]:=\sup _{\alpha \in \Lambda}\left\{-A^{\alpha}(x, y): D^{2} w-b^{\alpha}(x, y) \cdot \nabla w-f^{\alpha}(x, y)\right\}
$$

with uniformly continuous coefficients $a_{i j}=a_{j i}, b_{i}, f \in C\left(\bar{\Omega} \times \mathbb{R}^{n} \times \Lambda\right)$. It is assumed that $A^{\alpha}, b^{\alpha}, f^{\alpha}$ are $Y$-periodic in $y \in \mathbb{R}^{n}$ with respect to their second arguments and that the coefficients satisfy the Cordes condition (1.2) and the Lipschitz condition on $\bar{\Omega} \times \mathbb{R}^{n}$ uniformly in $\alpha \in \Lambda$.

It is well-known (see, e.g., Caffarelli, Souganidis, and Wang [7] and Evans [14, 15]) that the viscosity solution $u_{\varepsilon} \in C(\bar{\Omega})$ to (1.3) converges uniformly, as $\varepsilon \searrow 0$, to the viscosity solution $u_{0} \in C(\bar{\Omega})$ of the homogenized problem

$$
\left\{\begin{aligned}
u_{0}+H\left(x, \nabla u_{0}, D^{2} u_{0}\right) & =0 & & \text { in } \Omega, \\
u_{0} & =0 & & \text { on } \partial \Omega
\end{aligned}\right.
$$

for some function $H: \bar{\Omega} \times \mathbb{R}^{n} \times \mathcal{S}^{n \times n} \rightarrow \mathbb{R}$, the so-called effective Hamiltonian (here $\left.\mathcal{S}^{n \times n}:=\mathbb{R}_{\mathrm{sym}}^{n \times n}\right)$. The value of the effective Hamiltonian at a fixed point $(s, p, R) \in$ $\bar{\Omega} \times \mathbb{R}^{n} \times \mathcal{S}^{n \times n}$ can be obtained as the uniform limit of the sequence $\left\{-\sigma v^{\sigma}\right\}_{\sigma>0}$ as $\sigma \searrow 0$, where the so-called approximate corrector $v^{\sigma}=v^{\sigma}(\cdot ; s, p, R)$ is the solution to the problem

$$
\sigma v^{\sigma}+F\left[s, y, p, R+D_{y}^{2} v^{\sigma}\right]=0 \quad \text { for } y \in Y, \quad y \mapsto v^{\sigma}(y ; s, p, R) \text { is } Y \text {-periodic; }
$$

see, e.g., Alvarez and Bardi [2,3] and Camilli and Marchi [10]. Observe that the problem for the approximate corrector (1.5) fits into the framework of (1.1). For further homogenization results we refer to section 3 .

The main goal of this work is the efficient numerical approximation of the effective Hamiltonian. In order to do so, we first propose and analyze the numerical approximation of periodic boundary-value problems of the type (1.1) by a mixed finite element scheme (section 2) and then proceed with the numerical study of the approximate correctors and the effective Hamiltonian (section 3).

The motivation for studying the fully nonlinear second-order HJB equation comes from stochastic control theory for Markov diffusion processes and we refer the reader to Fleming and Soner [20]. Its study is a mathematically challenging task as there is no natural variational formulation and solvability has to be considered either in the sense of viscosity solutions (see Definition 3.3 and the user's guide by Crandall, Ishii, and Lions [13] for a comprehensive overview) or in the sense of strong solutions, i.e., functions admitting weak derivatives up to order two satisfying the equation pointwise almost everywhere. 
The finite element approximation of periodic HJB problems has not been studied a lot so far; the finite element approximation of the Dirichlet problem, however, has been the focus of active research over the past decade; see Feng, Glowinski, and Neilan [17] and Neilan, Salgado, and Zhang [31] for a survey of recent developments. The mixed finite element method presented in this paper is a modified version of the mixed scheme for the Dirichlet problem with Cordes coefficients introduced in the previous work by Gallistl and Süli [21], which enables the use of $H^{1}$-conforming finite elements. For further $H^{1}$-conforming finite element schemes, we refer to Camilli and Falcone [8], Camilli and Jakobsen [9], Jensen [25], and Jensen and Smears [26]. The first numerical scheme for HJB equations in the Cordes framework has been the discontinuous Galerkin finite element method in Smears and Süli [37, 38] (see also Smears and Süli [36]).

Numerical homogenization of second-order HJB problems, and nondivergenceform problems in general, has not been studied extensively so far. For the case of linear nondivergence-form equations, we refer the reader to the previous work Capdeboscq, Sprekeler, and Süli [11] (see also Sprekeler and Tran [39]) and to the references therein. For the case of second-order HJB equations, a finite difference scheme for the whole-space problem has been proposed in Camilli and Marchi [10]. In Finlay and Oberman [18, 19], the effective Hamiltonian is computed exactly for HJB operators of certain types and numerical simulations have been conducted. It seems that finite element schemes for the numerical homogenization of the problem (1.3) have not yet been constructed.

Let us note that there is a lot more work in the literature on the numerical approximation of the effective Hamiltonian arising in the homogenization of firstorder Hamilton-Jacobi equations; see various authors [1, 16, 22, 23, 30, 32, 33, 34]. The paper is structured as follows.

In section 2 , we propose and rigorously analyze a mixed finite element method for the approximation of the periodic solution to the HJB equation (1.1). We prove a priori (see Theorem 2.7) as well as a posteriori (see Theorem 2.9, Remark 2.10) error bounds with explicit error constants.

In section 3, we discuss the numerical homogenization of problems of the form (1.3). We provide the framework and theoretical homogenization results in sections 3.1 and 3.2 , respectively. We then analyze the approximation of the approximate corrector (1.5) by the mixed finite element scheme from section 2 and present a scheme for the approximation of the effective Hamiltonian in sections 3.3 and 3.4, respectively.

In section 4 , we present numerical experiments for the approximate corrector problems and the homogenized effective equation.

In section 5, we collect the proofs of the results contained in this work. Let us note that some proofs follow certain arguments of the earlier work [21]. Here, it is important to track the dependence of error constants in the Cordes parameters (see Remark 2.8), which is crucial for the arguments in sections 3.3 and 3.4. We have therefore included all details of the proofs.

For simplicity, all results in this work are presented for dimensions $n \in\{2,3\}$ in which we define the rotation (curl) of a sufficiently regular vector field $w=\left(w_{i}\right)_{1 \leq i \leq n}$ : $\mathbb{R}^{n} \rightarrow \mathbb{R}^{n}$ by

$$
\begin{aligned}
\operatorname{rot}(w):=\partial_{2} w_{1}-\partial_{1} w_{2} & \text { if } n=2, \\
\operatorname{rot}(w):=\left(\partial_{2} w_{3}-\partial_{3} w_{2}, \partial_{3} w_{1}-\partial_{1} w_{3}, \partial_{1} w_{2}-\partial_{2} w_{1}\right) & \text { if } n=3 .
\end{aligned}
$$

The results in this paper remain valid for higher dimensions $n \geq 4$, in which case the rotation operator needs to be replaced by the exterior derivative operator. 


\section{Mixed FEM for periodic HJB problems.}

2.1. Framework. In dimension $n \in\{2,3\}$, we let $Y:=(0,1)^{n}$ denote the unit cell in $\mathbb{R}^{n}$. Further, we let $\Lambda$ be a compact metric space. We then consider the problem of finding periodic strong solutions to the second-order HJB equation

$$
\sup _{\alpha \in \Lambda}\left\{-A^{\alpha}: D^{2} u-b^{\alpha} \cdot \nabla u+c^{\alpha} u-f^{\alpha}\right\}=0 \quad \text { in } Y, \quad u \in H_{\mathrm{per}}^{2}(Y),
$$

where we make the following assumptions on the coefficients: Writing $\mathcal{S}^{n \times n} \subset \mathbb{R}^{n \times n}$ for the space of real symmetric $n \times n$ matrices, we assume that the functions

$$
\begin{aligned}
& A=\left(a_{i j}\right)_{1 \leq i, j \leq n}: \mathbb{R}^{n} \times \Lambda \rightarrow \mathcal{S}^{n \times n}, \quad(y, \alpha) \mapsto A(y, \alpha)=: A^{\alpha}(y), \\
& b=\left(b_{i}\right)_{1 \leq i \leq n}: \mathbb{R}^{n} \times \Lambda \rightarrow \mathbb{R}^{n}, \quad(y, \alpha) \mapsto b(y, \alpha)=: b^{\alpha}(y), \\
& c: \mathbb{R}^{n} \times \Lambda \rightarrow \mathbb{R}, \quad(y, \alpha) \mapsto c(y, \alpha)=: c^{\alpha}(y), \\
& f: \mathbb{R}^{n} \times \Lambda \rightarrow \mathbb{R}, \quad(y, \alpha) \mapsto f(y, \alpha)=: f^{\alpha}(y)
\end{aligned}
$$

are $Y$-periodic in $y \in \mathbb{R}^{n}$ and uniformly continuous, i.e., $a_{i j}, b_{i}, c, f \in C\left(\mathbb{R}^{n} \times \Lambda\right)$. Further, we assume that the zeroth-order coefficient is positive,

$$
\inf _{\mathbb{R}^{n} \times \Lambda} c>0,
$$

that the matrix-valued function $A$ is uniformly elliptic,

$$
\exists \zeta_{1}, \zeta_{2}>0: \quad \zeta_{1}|\xi|^{2} \leq A(y, \alpha) \xi \cdot \xi \leq \zeta_{2}|\xi|^{2} \quad \forall y, \xi \in \mathbb{R}^{n}, \alpha \in \Lambda,
$$

and that the Cordes condition

$$
|A|^{2}+\frac{|b|^{2}}{2 \lambda}+\frac{c^{2}}{\lambda^{2}} \leq \frac{1}{n+\delta}\left(\operatorname{tr}(A)+\frac{c}{\lambda}\right)^{2} \quad \text { in } \mathbb{R}^{n} \times \Lambda
$$

holds for some constants $\lambda>0$ and $\delta \in(0,1)$. The Cordes condition arises naturally for stochastic control problems (see [37, Example 1]) and we refer the reader to [37] for a brief discussion of the Cordes condition.

Let us introduce the function $\gamma \in C\left(\mathbb{R}^{n} \times \Lambda\right)$ defined by

$$
\gamma:=\left(|A|^{2}+\frac{|b|^{2}}{2 \lambda}+\frac{c^{2}}{\lambda^{2}}\right)^{-1}\left(\operatorname{tr}(A)+\frac{c}{\lambda}\right) .
$$

We let $\gamma^{\alpha}: \mathbb{R}^{n} \rightarrow \mathbb{R}, \gamma^{\alpha}(y):=\gamma(y, \alpha)$ for $\alpha \in \Lambda$ and consider the renormalized HJB equation

$$
\sup _{\alpha \in \Lambda}\left\{\gamma^{\alpha}\left(-A^{\alpha}: D^{2} u-b^{\alpha} \cdot \nabla u+c^{\alpha} u-f^{\alpha}\right)\right\}=0 \quad \text { in } Y, \quad u \in H_{\mathrm{per}}^{2}(Y) .
$$

The function $\gamma$ takes the role of a (positive) multiplying factor for the equation; note that $\inf _{\mathbb{R}^{n} \times \Lambda} \gamma>0$. Hence $u \in H_{\text {per }}^{2}(Y)$ is a solution of (2.1) if, and only if, it is a solution of $(2.5)$.

It can then be shown that the problem (2.1) is well-posed in the sense of strong solutions, following the steps of the proof of [37, Theorem 3] (note the sign-difference in the coefficient functions). The proof is omitted.

THEOREM 2.1 (well-posedness). In the situation described above, there exists a unique strong solution $u \in H_{\mathrm{per}}^{2}(Y)$ to the problem (2.1). Further, $u$ is also the unique strong solution to the problem (2.5). 
2.2. Mixed formulation of the problem. We construct a mixed finite element method for the numerical approximation of the strong periodic solution to (2.1) similarly to the scheme presented in [21]. The mixed formulation relies on rewriting the problem (2.5) as

$$
\sup _{\alpha \in \Lambda}\left\{\gamma^{\alpha}\left(-A^{\alpha}: D w-b^{\alpha} \cdot \nabla u+c^{\alpha} u-f^{\alpha}\right)\right\}=0, \quad w=\nabla u .
$$

We denote the space of functions $v \in H_{\mathrm{per}}^{1}(Y)$ with zero mean over the unit cell $Y$ by

$$
W_{\text {per }}(Y):=\left\{v \in H_{\text {per }}^{1}(Y): \int_{Y} v=0\right\} .
$$

We further let $W_{\text {per }}\left(Y ; \mathbb{R}^{n}\right):=\left(W_{\text {per }}(Y)\right)^{n}$ and denote the Jacobian of a function $w \in W_{\text {per }}\left(Y ; \mathbb{R}^{n}\right)$ by $D w$. Noting that $Y=(0,1)^{n}$ is convex and $\operatorname{diam}(Y)=\sqrt{n}$, we have the Poincaré inequality (see $[4$, Theorem 3.2]) for scalar functions,

$$
\|v\|_{L^{2}(Y)} \leq \frac{\sqrt{n}}{\pi}\|\nabla v\|_{L^{2}(Y)} \quad \forall v \in W_{\text {per }}(Y),
$$

and the corresponding inequality for vector-valued functions,

$$
\|w\|_{L^{2}(Y)} \leq \frac{\sqrt{n}}{\pi}\|D w\|_{L^{2}(Y)} \quad \forall w \in W_{\mathrm{per}}\left(Y ; \mathbb{R}^{n}\right) .
$$

Noting that a solution $u \in H_{\text {per }}^{2}(Y)$ to $(2.6)$ satisfies $w=\nabla u \in W_{\text {per }}\left(Y ; \mathbb{R}^{n}\right)$, we define the function space

$$
X:=W_{\text {per }}\left(Y ; \mathbb{R}^{n}\right) \times H_{\text {per }}^{1}(Y) .
$$

Further, we let $M \subset W_{\text {per }}(Y)$ be a closed linear subspace. Admissible choices include $M=\{0\}$ and $M=W_{\text {per }}(Y)$. We remark that $M=\{0\}$ not only simplifies the theory, but also leads to positive definite linearized systems and is therefore favorable from the point of view of numerical linear algebra. It was, however, experimentally observed in [21] that a nontrivial choice of $M_{h}$ (see section 2.4) may have a positive effect on the iteration numbers in a semismooth Newton iteration. We therefore allow for some freedom regarding the choices of $M$ and $M_{h}$ in our theory.

The mixed formulation is defined as the following problem: Find $m \in M$ and $(w, u) \in X$ such that

$$
\left\{\begin{aligned}
a\left((w, u),\left(w^{\prime}, u^{\prime}\right)\right)+b\left(m,\left(w^{\prime}, u^{\prime}\right)\right) & =0 & & \forall\left(w^{\prime}, u^{\prime}\right) \in X, \\
b\left(m^{\prime},(w, u)\right) & =0 & & \forall m^{\prime} \in M,
\end{aligned}\right.
$$

where the semilinear form $a: X \times X \rightarrow \mathbb{R}$ is given by

$$
\begin{aligned}
& a\left((w, u),\left(w^{\prime}, u^{\prime}\right)\right) \\
& :=\int_{Y} F_{\gamma}[(w, u)] L_{\lambda}\left(w^{\prime}, u^{\prime}\right)+\sigma_{1} \int_{Y} \operatorname{rot}(w) \cdot \operatorname{rot}\left(w^{\prime}\right)+\sigma_{2} \int_{Y}(\nabla u-w) \cdot\left(\nabla u^{\prime}-w^{\prime}\right),
\end{aligned}
$$

and the bilinear form $b: M \times X \rightarrow \mathbb{R}$ is given by

$$
b(m,(w, u)):=\int_{Y} \nabla m \cdot(\nabla u-w)
$$


for $(w, u),\left(w^{\prime}, u^{\prime}\right) \in X$ and $m \in M$. Here, we have used the operators

$$
\begin{aligned}
F_{\gamma}[(w, u)] & :=\sup _{\alpha \in \Lambda}\left\{\gamma^{\alpha}\left(-A^{\alpha}: D w-b^{\alpha} \cdot \nabla u+c^{\alpha} u-f^{\alpha}\right)\right\}, \\
L_{\lambda}(w, u) & :=-\nabla \cdot w+\lambda u,
\end{aligned}
$$

acting on $(w, u) \in X$, and the positive constants

$$
\begin{aligned}
& \sigma_{1}:=\sigma_{1}(\delta):=1-\frac{1}{2} \sqrt{1-\delta} \\
& \sigma_{2}:=\lambda \tilde{\sigma}_{2}(\delta):=\lambda\left(\frac{1-\sqrt{1-\delta}}{2}+\frac{1}{4(1-\sqrt{1-\delta})}\right) .
\end{aligned}
$$

We proceed by showing well-posedness of this mixed formulation.

2.3. Well-posedness of the mixed formulation. We define a norm on the function space $X=W_{\text {per }}\left(Y ; \mathbb{R}^{n}\right) \times H_{\text {per }}^{1}(Y)$ by

$$
\|(w, u)\|_{\lambda}^{2}:=\|D w\|_{L^{2}(Y)}^{2}+2 \lambda\|\nabla u\|_{L^{2}(Y)}^{2}+\lambda^{2}\|u\|_{L^{2}(Y)}^{2}, \quad(w, u) \in X .
$$

It is easy to verify that this does indeed define a norm on $X$. We observe that there holds

$$
\|D w\|_{L^{2}(Y)}^{2}=\|\operatorname{rot}(w)\|_{L^{2}(Y)}^{2}+\|\nabla \cdot w\|_{L^{2}(Y)}^{2} \quad \forall w \in H_{\mathrm{per}}^{1}\left(Y ; \mathbb{R}^{n}\right),
$$

which follows from the formal calculation (using integration by parts twice)

$$
\int_{Y}|D w|^{2}-\int_{Y}|\operatorname{rot}(w)|^{2}=\sum_{i, j=1}^{n} \int_{Y} \partial_{i} w_{j} \partial_{j} w_{i}=\sum_{i, j=1}^{n} \int_{Y} \partial_{i} w_{i} \partial_{j} w_{j}=\int_{Y}|\nabla \cdot w|^{2}
$$

and a density argument. Note that compared to the usual Maxwell-type inequality [12], we have obtained the equality (2.10) thanks to periodicity. Next, we derive two preliminary estimates.

Lemma 2.2 (preliminary estimates). Let $(w, u),\left(w^{\prime}, u^{\prime}\right) \in X$ and $\rho \in(0,2)$. Then, there holds

$$
\begin{gathered}
\left\|F_{\gamma}[(w, u)]-F_{\gamma}\left[\left(w^{\prime}, u^{\prime}\right)\right]-L_{\lambda}\left(w-w^{\prime}, u-u^{\prime}\right)\right\|_{L^{2}(Y)} \\
\leq \sqrt{1-\delta}\left\|\left(w-w^{\prime}, u-u^{\prime}\right)\right\|_{\lambda},
\end{gathered}
$$

and we have the Miranda-Talenti-type estimate

$$
\frac{2-\rho}{2}\|(w, u)\|_{\lambda}^{2} \leq\|\operatorname{rot}(w)\|_{L^{2}(Y)}^{2}+\left\|L_{\lambda}(w, u)\right\|_{L^{2}(Y)}^{2}+\frac{\lambda}{\rho}\|\nabla u-w\|_{L^{2}(Y)}^{2} .
$$

With these estimates in hand, we can proceed with showing essential properties of the maps $a$ and $b$, including monotonicity, Lipschitz continuity, and an inf-sup condition, which will allow us to show the well-posedness of the mixed formulation.

LEMMA 2.3 (monotonicity, Lipschitz continuity, and inf-sup condition). We have the following properties:

(i) Monotonicity: For any $(w, u),\left(w^{\prime}, u^{\prime}\right) \in X$, we have

$$
\begin{aligned}
& C_{M}\left\|\left(w-w^{\prime}, u-u^{\prime}\right)\right\|_{\lambda}^{2} \\
& \quad \leq a\left((w, u),\left(w-w^{\prime}, u-u^{\prime}\right)\right)-a\left(\left(w^{\prime}, u^{\prime}\right),\left(w-w^{\prime}, u-u^{\prime}\right)\right)
\end{aligned}
$$

with the monotonicity constant $C_{M}:=\frac{1}{4}(1-\sqrt{1-\delta})>0$. 
(ii) Lipschitz continuity: For any $(w, u),\left(w^{\prime}, u^{\prime}\right),(z, v) \in X$, we have

$$
\left|a((w, u),(z, v))-a\left(\left(w^{\prime}, u^{\prime}\right),(z, v)\right)\right| \leq C_{L}\left\|\left(w-w^{\prime}, u-u^{\prime}\right)\right\|_{\lambda}\|(z, v)\|_{\lambda}
$$

with the Lipschitz constant $C_{L}:=2+\sqrt{2} \sqrt{1-\delta}+\sigma_{1}(\delta)+\tilde{\sigma}_{2}(\delta)\left(\frac{1}{2}+\frac{n}{\pi^{2}} \lambda\right)>0$.

(iii) Inf-sup condition: We have

$$
\inf _{m^{\prime} \in M \backslash\{0\}} \sup _{\left(w^{\prime}, u^{\prime}\right) \in X \backslash\{0\}} \frac{b\left(m^{\prime},\left(w^{\prime}, u^{\prime}\right)\right)}{\left\|\nabla m^{\prime}\right\|_{L^{2}(Y)}\left\|\left(w^{\prime}, u^{\prime}\right)\right\|_{\lambda}} \geq c_{b}
$$

with the inf-sup constant $c_{b}:=\lambda^{-\frac{1}{2}}\left(2+\frac{n}{\pi^{2}} \lambda\right)^{-\frac{1}{2}}>0$.

Remark 2.4 (local Lipschitz estimate). Similarly, one obtains the local Lipschitz estimate

$$
\begin{aligned}
& \left|a_{I}((w, u),(z, v))-a_{I}\left(\left(w^{\prime}, u^{\prime}\right),(z, v)\right)\right| \\
& \quad \leq C_{L}^{\prime}\left(\left\|\mid\left(w-w^{\prime}, u-u^{\prime}\right)\right\|_{\lambda, I}+\left\|w-w^{\prime}\right\|_{L^{2}(I)}\right)\left(\|(z, v)\|\left\|_{\lambda, I}+\right\| z \|_{L^{2}(I)}\right)
\end{aligned}
$$

for all $(w, u),\left(w^{\prime}, u^{\prime}\right),(z, v) \in X$ and any open $I \subset Y$ with a constant $C_{L}^{\prime}=C_{L}^{\prime}(\delta, \lambda, n)>$ 0 . Here, the subscript $I$ in $a_{I}$ and $\|\cdot \cdot\|_{\lambda, I}$ denotes that integrals in the corresponding definitions are taken over the set $I$.

We note that the inf-sup condition (2.14) is trivially satisfied when $M=\{0\}$ as $\inf \emptyset:=\infty$. Now we are in a position to state the well-posedness of the mixed formulation, i.e., the existence and uniqueness of a solution $(m,(w, u)) \in M \times X$ to (2.9).

TheOrem 2.5 (well-posedness of the mixed formulation). The mixed formulation (2.9) admits a unique solution $(m,(w, u)) \in M \times X$. Further, $m=0, u \in H_{\mathrm{per}}^{2}(Y)$ with $\nabla u=w$ and $u$ is the solution to (2.1).

2.4. The discrete mixed formulation. We take closed linear subspaces $W_{h} \subset$ $W_{\text {per }}\left(Y ; \mathbb{R}^{n}\right), U_{h} \subset H_{\text {per }}^{1}(Y), M_{h} \subset U_{h} \cap M$ (recall that $M \subset W_{\text {per }}(Y)$ ) and define

$$
X_{h}:=W_{h} \times U_{h} \subset X .
$$

We then define the discrete mixed formulation as the following problem: Find $m_{h} \in$ $M_{h}$ and $\left(w_{h}, u_{h}\right) \in X_{h}$ such that

$$
\left\{\begin{aligned}
a\left(\left(w_{h}, u_{h}\right),\left(w_{h}^{\prime}, u_{h}^{\prime}\right)\right)+b\left(m_{h},\left(w_{h}^{\prime}, u_{h}^{\prime}\right)\right)=0 & \forall\left(w_{h}^{\prime}, u_{h}^{\prime}\right) \in X_{h}, \\
b\left(m_{h}^{\prime},\left(w_{h}, u_{h}\right)\right)=0 & \forall m_{h}^{\prime} \in M_{h} .
\end{aligned}\right.
$$

We note that we have the boundedness of $b$ and a discrete inf-sup condition.

LEMma 2.6 (boundedness of the bilinear form $b$ and discrete inf-sup condition). For any $\left(m^{\prime},\left(w^{\prime}, u^{\prime}\right)\right) \in M \times X$, we have

$$
b\left(m^{\prime},\left(w^{\prime}, u^{\prime}\right)\right) \leq C_{b}\left\|\nabla m^{\prime}\right\|_{L^{2}(Y)}\left\|\left(w^{\prime}, u^{\prime}\right)\right\|_{\lambda}
$$

with the constant $C_{b}:=\lambda^{-\frac{1}{2}}\left(\frac{1}{2}+\frac{n}{\pi^{2}} \lambda\right)^{\frac{1}{2}}>0$. Further, the discrete inf-sup condition

$$
\inf _{m_{h}^{\prime} \in M_{h} \backslash\{0\}} \sup _{\left(w_{h}^{\prime}, u_{h}^{\prime}\right) \in X_{h} \backslash\{0\}} \frac{b\left(m_{h}^{\prime},\left(w_{h}^{\prime}, u_{h}^{\prime}\right)\right)}{\left\|\nabla m_{h}^{\prime}\right\|_{L^{2}(Y)}\left\|\left(w_{h}^{\prime}, u_{h}^{\prime}\right)\right\|_{\lambda}} \geq c_{b}
$$

holds with $c_{b}>0$ as in Lemma 2.3(iii). 
It follows that we have the well-posedness of the discrete mixed formulation analogously to Theorem 2.5. We also obtain an error bound.

THEOREM 2.7 (well-posedness and error bound). There exists a unique solution $\left(m_{h},\left(w_{h}, u_{h}\right)\right) \in M_{h} \times X_{h}$ to the discrete mixed formulation (2.15). Further, we have

$$
\left\|\left(w-w_{h}, u-u_{h}\right)\right\|_{\lambda} \leq C_{e} \inf _{\left(w_{h}^{\prime}, u_{h}^{\prime}\right) \in X_{h}}\left\|\left(w-w_{h}^{\prime}, u-u_{h}^{\prime}\right)\right\|_{\lambda},
$$

where $(m,(w, u)) \in M \times X$ denotes the solution to $(2.9)$ and $C_{e}=C_{e}(\delta, \lambda, n)>0$ is the constant

$$
C_{e}:=2 \frac{C_{L}}{C_{M}}\left(1+\frac{C_{b}}{c_{b}}\right)
$$

with $C_{L}, C_{M}, C_{b}, c_{b}>0$ from Lemmata 2.3 and 2.6.

Remark 2.8. Note that the error constant $C_{e}=C_{e}(\delta, \lambda, n)$ is monotonically increasing in $\lambda$.

Besides this a priori error bound, the monotonicity property from Lemma 2.3 allows us to obtain an a posteriori error bound.

Theorem 2.9 (a posteriori error bound and efficiency). For the solution

$$
(m,(w, u)) \in M \times X
$$

to the mixed formulation (2.9) and the solution $\left(m_{h},\left(w_{h}, u_{h}\right)\right) \in M_{h} \times X_{h}$ to the discrete mixed formulation (2.15), we have the error bound

$$
\begin{aligned}
& \left\|\left(w-w_{h}, u-u_{h}\right)\right\|_{\lambda}^{2} \\
& \leq 2 C_{M}^{-1}\left(C_{M}^{-1}\left\|F_{\gamma}\left[\left(w_{h}, u_{h}\right)\right]\right\|_{L^{2}(Y)}^{2}+\sigma_{1}\left\|\operatorname{rot}\left(w_{h}\right)\right\|_{L^{2}(Y)}^{2}+\sigma_{2}\left\|w_{h}-\nabla u_{h}\right\|_{L^{2}(Y)}^{2}\right)
\end{aligned}
$$

and the efficiency estimate

$$
\begin{gathered}
\frac{1}{2}\left\|F_{\gamma}\left[\left(w_{h}, u_{h}\right)\right]\right\|_{L^{2}(Y)}^{2}+\sigma_{1}\left\|\operatorname{rot}\left(w_{h}\right)\right\|_{L^{2}(Y)}^{2}+\sigma_{2}\left\|w_{h}-\nabla u_{h}\right\|_{L^{2}(Y)}^{2} \\
\leq\left(C_{L}+\frac{1-\delta}{2}\right)\left\|\left(w-w_{h}, u-u_{h}\right)\right\|_{\lambda}^{2},
\end{gathered}
$$

where $C_{M}, C_{L}>0$ are the constants from Lemma 2.3 .

Remark 2.10 (local efficiency). Similarly, one obtains the local efficiency estimate

$$
\begin{aligned}
\frac{1}{2}\left\|F_{\gamma}\left[\left(w_{h}, u_{h}\right)\right]\right\|_{L^{2}(I)}^{2} & +\sigma_{1}\left\|\operatorname{rot}\left(w_{h}\right)\right\|_{L^{2}(I)}^{2}+\sigma_{2}\left\|w_{h}-\nabla u_{h}\right\|_{L^{2}(I)}^{2} \\
\leq & \left(2 C_{L}^{\prime}+\frac{1-\delta}{2}\right)\left(\left\|\left(w-w_{h}, u-u_{h}\right)\right\|_{\lambda, I}^{2}+\left\|w-w_{h}\right\|_{L^{2}(I)}^{2}\right)
\end{aligned}
$$

for any open $I \subset Y$, where $C_{L}^{\prime}>0$ is the constant from Remark 2.4.

\section{Numerical homogenization of HJB equations.}

3.1. Framework. Let $\Omega \subset \mathbb{R}^{n}$ be a bounded convex domain in dimension $n \in$ $\{2,3\}$ and let $\Lambda$ be a compact metric space. For $\varepsilon>0$ small, we consider problems of the form

$$
\left\{\begin{aligned}
\sup _{\alpha \in \Lambda}\left\{-A^{\alpha}\left(\cdot, \frac{\cdot}{\varepsilon}\right): D^{2} u_{\varepsilon}-b^{\alpha}\left(\cdot, \frac{\cdot}{\varepsilon}\right) \cdot \nabla u_{\varepsilon}+u_{\varepsilon}-f^{\alpha}\left(\cdot, \frac{\cdot}{\varepsilon}\right)\right\}=0 & \text { in } \Omega \\
u_{\varepsilon}=0 & \text { on } \partial \Omega
\end{aligned}\right.
$$


where we assume that the functions

$$
\begin{array}{rlrl}
A=\left(a_{i j}\right)_{1 \leq i, j \leq n}: \bar{\Omega} \times \mathbb{R}^{n} \times \Lambda & \rightarrow \mathcal{S}^{n \times n}, & & (x, y, \alpha) \mapsto A(x, y, \alpha)=: A^{\alpha}(x, y), \\
b=\left(b_{i}\right)_{1 \leq i \leq n}: \bar{\Omega} \times \mathbb{R}^{n} \times \Lambda \rightarrow \mathbb{R}^{n}, & & (x, y, \alpha) \mapsto b(x, y, \alpha)=: b^{\alpha}(x, y), \\
f: \bar{\Omega} \times \mathbb{R}^{n} \times \Lambda \rightarrow \mathbb{R}, & & (x, y, \alpha) \mapsto f(x, y, \alpha)=: f^{\alpha}(x, y)
\end{array}
$$

satisfy the following assumptions:

(i) Continuity: $A, b, f$ are continuous on $\bar{\Omega} \times \mathbb{R}^{n} \times \Lambda$.

(ii) Periodicity: $A^{\alpha}(x, \cdot), b^{\alpha}(x, \cdot), f^{\alpha}(x, \cdot)$ are $Y$-periodic for fixed $\alpha \in \Lambda$ and $x \in \bar{\Omega}$.

(iii) Regularity: $A^{\alpha}, b^{\alpha}, f^{\alpha}$ are Lipschitz on $\bar{\Omega} \times \mathbb{R}^{n}$ uniformly in $\alpha \in \Lambda$.

(iv) Ellipticity: There exist $\zeta_{1}, \zeta_{2}>0$ such that $\zeta_{1}|\xi|^{2} \leq A \xi \cdot \xi \leq \zeta_{2}|\xi|^{2}$ in $\bar{\Omega} \times$ $\mathbb{R}^{n} \times \Lambda$ for all $\xi \in \mathbb{R}^{n}$.

Further, it is assumed that the Cordes condition

$$
|A|^{2}+\frac{|b|^{2}}{2 \lambda}+\frac{1}{\lambda^{2}} \leq \frac{1}{n+\delta}\left(\operatorname{tr}(A)+\frac{1}{\lambda}\right)^{2} \quad \text { in } \bar{\Omega} \times \mathbb{R}^{n} \times \Lambda
$$

holds for some constants $\lambda>0$ and $\delta \in(0,1)$. Then, we have well-posedness in the sense of strong solutions; see [37].

THEOREM 3.1 (existence and uniqueness of strong solutions). In this situation, for any given $\varepsilon>0$, there exists a unique strong solution $u_{\varepsilon} \in H^{2}(\Omega) \cap H_{0}^{1}(\Omega)$ to (3.1).

Remark 3.2. Problems involving a nonconstant zeroth-order coefficient, i.e., problems of the form

$$
\begin{aligned}
\sup _{\alpha \in \Lambda}\left\{-A^{\alpha}\left(\cdot, \frac{\cdot}{\varepsilon}\right): D^{2} v_{\varepsilon}-b^{\alpha}\left(\cdot, \frac{\cdot}{\varepsilon}\right) \cdot \nabla v_{\varepsilon}+c^{\alpha}\left(\cdot, \frac{\cdot}{\varepsilon}\right) v_{\varepsilon}-f^{\alpha}\left(\cdot, \frac{\cdot}{\varepsilon}\right)\right\}=0 & \text { in } \Omega, \\
v_{\varepsilon}=0 & \text { on } \partial \Omega
\end{aligned}
$$

with $c^{\alpha}$ satisfying the same assumptions as the components of $b^{\alpha}$, and additionally $\inf _{\bar{\Omega} \times \mathbb{R}^{n} \times \Lambda} c>0$, can be reduced to a problem of the form (3.1). This is due to the fact that division by $c^{\alpha}(x, x / \varepsilon)$ inside the argument of the supremum does not change the sets of strong and viscosity solutions; see [27, Remark 2.2].

3.2. Homogenization. In this section, we briefly recall known homogenization results from the literature. Let us start by recalling one of the several equivalent definitions of a viscosity solution; see [28].

Definition 3.3 (viscosity solution). Let $\Omega \subset \mathbb{R}^{n}$ be open and $F: \Omega \times \mathbb{R} \times \mathbb{R}^{n} \times$ $\mathcal{S}^{n \times n} \rightarrow \mathbb{R}$ be continuous. A continuous function $u: \Omega \rightarrow \mathbb{R}, u \in C(\bar{\Omega})$, is called a viscosity solution to the equation

$$
F\left(x, u, \nabla u, D^{2} u\right)=0 \quad \text { in } \Omega
$$

if for any $\phi \in C^{2}(\Omega)$ there holds

$x_{0} \in \Omega$ local maximum point of $u-\phi \Longrightarrow F\left(x_{0}, u\left(x_{0}\right), \nabla \phi\left(x_{0}\right), D^{2} \phi\left(x_{0}\right)\right) \leq 0$,

$x_{0} \in \Omega$ local minimum point of $u-\phi \Longrightarrow F\left(x_{0}, u\left(x_{0}\right), \nabla \phi\left(x_{0}\right), D^{2} \phi\left(x_{0}\right)\right) \geq 0$.

For an overview of the theory of viscosity solutions for second-order equations we refer the reader to [13]. Note that the strong solution $u_{\varepsilon} \in H^{2}(\Omega) \cap H_{0}^{1}(\Omega)$ to (3.1) 
belongs to $C(\bar{\Omega})$ in dimensions $n \in\{2,3\}$ and a natural question to ask is whether $u_{\varepsilon}$ is a viscosity solution. In fact, it is known that if one has regularity $u_{\varepsilon} \in W_{\text {loc }}^{2, n}(\Omega)$, then $u_{\varepsilon}$ is a viscosity solution to (3.1); see [6, 28, 29]. We also note that the viscosity solution to (3.1) is unique; see [24]. We then have the following result; see [35].

Remark 3.4 (regularity). Let $u_{\varepsilon} \in H^{2}(\Omega) \cap H_{0}^{1}(\Omega)$ be the unique strong solution to (3.1) given by Theorem 3.1. Then

$$
u_{\varepsilon} \in C^{2, \tilde{\alpha}}(\Omega) \cap C(\bar{\Omega})
$$

for some $\tilde{\alpha}>0$ and $u_{\varepsilon}$ is the unique viscosity solution to (3.1). Further, if $\partial \Omega \in C^{2, \beta}$ for some $\beta>0$, then $u_{\varepsilon} \in C^{2, \tilde{\alpha}}(\bar{\Omega})$ for some $\tilde{\alpha}>0$.

With this observation in hand, we can use the well-known homogenization results for viscosity solutions; see $[7,14,15]$. Before stating the convergence theorem, let us introduce the effective Hamiltonian

$$
H: \bar{\Omega} \times \mathbb{R}^{n} \times \mathcal{S}^{n \times n} \rightarrow \mathbb{R},
$$

defined as follows. For given $(s, p, R) \in \bar{\Omega} \times \mathbb{R}^{n} \times \mathcal{S}^{n \times n}$ we define $H(s, p, R) \in \mathbb{R}$ to be the unique real number such that there exists a function $v=v(\cdot ; s, p, R) \in C\left(\mathbb{R}^{n}\right)$, a so-called corrector, that is a viscosity solution to

$$
\sup _{\alpha \in \Lambda}\left\{-A_{s}^{\alpha}: D^{2} v-g_{s, p, R}^{\alpha}\right\}=H(s, p, R) \quad \text { in } \mathbb{R}^{n}, \quad v(\cdot ; s, p, R) \text { is } Y \text {-periodic, }
$$

where $A_{s}^{\alpha}(y):=A^{\alpha}(s, y)$ and $g_{s, p, R}^{\alpha}(y):=A^{\alpha}(s, y): R+b^{\alpha}(s, y) \cdot p+f^{\alpha}(s, y)$ for $y \in \mathbb{R}^{n}, \alpha \in \Lambda$.

THEOREM 3.5 (homogenization of HJB problems). With the effective Hamiltonian $H: \bar{\Omega} \times \mathbb{R}^{n} \times \mathcal{S}^{n \times n} \rightarrow \mathbb{R}$ defined as above, the solution $u_{\varepsilon}$ to (3.1) converges uniformly on $\bar{\Omega}$ to the viscosity solution $u_{0} \in C(\bar{\Omega})$ of the problem

$$
\left\{\begin{array}{rlrl}
u_{0}+H\left(x, \nabla u_{0}, D^{2} u_{0}\right) & =0 & & \text { in } \Omega, \\
u_{0}=0 & & \text { on } \partial \Omega .
\end{array}\right.
$$

We call (3.4) the homogenized (or effective) problem corresponding to the HJB problem (3.1).

Let us note that rates for the convergence of $u_{\varepsilon}$ to the homogenized solution $u_{0}$ have been derived for the whole space problem in [10].

The effective Hamiltonian can also be obtained through a limit of ergodic approximations, the so-called approximate correctors; see [3] and the references therein. For $(s, p, R) \in \bar{\Omega} \times \mathbb{R}^{n} \times \mathcal{S}^{n \times n}$ and $\sigma>0$, the approximate corrector $v^{\sigma}=v^{\sigma}(\cdot ; s, p, R) \in$ $C\left(\mathbb{R}^{n}\right)$ is defined to be the viscosity solution to

$$
\sigma v^{\sigma}+\sup _{\alpha \in \Lambda}\left\{-A_{s}^{\alpha}: D^{2} v^{\sigma}-g_{s, p, R}^{\alpha}\right\}=0 \quad \text { in } \mathbb{R}^{n}, \quad v^{\sigma}(\cdot ; s, p, R) \text { is } Y \text {-periodic. }
$$

Remark 3.6 (regularity of approximate correctors). The viscosity solution $v^{\sigma}=$ $v^{\sigma}(\cdot ; s, p, R) \in C\left(\mathbb{R}^{n}\right)$ to $(3.5)$ is in fact a classical solution $v^{\sigma}(\cdot ; s, p, R) \in C^{2}\left(\mathbb{R}^{n}\right)$. Further, there exists $\tilde{\alpha} \in(0,1)$ such that

$$
\left\|\sigma v^{\sigma}(\cdot ; s, p, R)\right\|_{C\left(\mathbb{R}^{n}\right)}+\left\|v^{\sigma}(\cdot ; s, p, R)-v^{\sigma}(0 ; s, p, R)\right\|_{C^{2, \tilde{\alpha}}\left(\mathbb{R}^{n}\right)} \lesssim 1+|p|+|R|
$$

for all $(s, p, R) \in \bar{\Omega} \times \mathbb{R}^{n} \times \mathcal{S}^{n \times n}$; see $[2,10]$. 
The value $H(s, p, R) \in \mathbb{R}$ for the effective Hamiltonian at the point $(s, p, R)$ is then the uniform limit of the sequence $\left\{-\sigma v^{\sigma}\right\}_{\sigma>0}$ as $\sigma \rightarrow 0$; see [10].

Lemma 3.7 (properties of the effective Hamiltonian). The following statements hold true.

(i) The sequence $\left\{-\sigma v^{\sigma}(\cdot ; s, p, R)\right\}_{\sigma>0}$ converges uniformly to the constant value $H(s, p, R)$ with

$$
\left\|-\sigma v^{\sigma}(\cdot ; s, p, R)-H(s, p, R)\right\|_{\infty} \lesssim \sigma(1+|p|+|R|)
$$

for all $(s, p, R) \in \bar{\Omega} \times \mathbb{R}^{n} \times \mathcal{S}^{n \times n}$ and $\sigma>0$ sufficiently small.

(ii) The effective Hamiltonian $H=H(s, p, R)$ is uniformly elliptic, it is convex in $R$, and we have

$$
\begin{aligned}
\left|H\left(s, p_{1}, R_{1}\right)-H\left(s, p_{2}, R_{2}\right)\right| & \lesssim\left|p_{1}-p_{2}\right|+\left|R_{1}-R_{2}\right|, \\
\left|H\left(s_{1}, p, R\right)-H\left(s_{2}, p, R\right)\right| & \lesssim\left|s_{1}-s_{2}\right|(1+|p|+|R|)
\end{aligned}
$$

for any $s, s_{1}, s_{2} \in \bar{\Omega}, p, p_{1}, p_{2} \in \mathbb{R}^{n}$, and $R, R_{1}, R_{2} \in \mathcal{S}^{n \times n}$.

Note that the properties of the approximate correctors from Remark 3.6 and Lemma 3.7(i) allow passage to the limit $\sigma \rightarrow 0$ in (3.5) and guarantee the existence of a corrector $v(\cdot ; s, p, R) \in C^{2}\left(\mathbb{R}^{n}\right)$ (i.e., a classical solution to (3.3)). We also note that the properties of the effective Hamiltonian from Lemma 3.7(ii) yield a regularity result for the homogenized solution as it is of the type of problems studied in [35].

Remark 3.8 (regularity of the homogenized solution). The viscosity solution $u_{0} \in C(\bar{\Omega})$ to the homogenized problem (3.4) satisfies

$$
u_{0} \in C^{2, \tilde{\alpha}}(\Omega) \cap C(\bar{\Omega})
$$

for some $\tilde{\alpha}>0$. Further, if $\partial \Omega \in C^{2, \beta}$ for some $\beta>0$, then $u_{0} \in C^{2, \tilde{\alpha}}(\bar{\Omega})$ for some $\tilde{\alpha}>0$.

3.3. Approximation of the approximate corrector. We construct a mixed finite element method for the numerical approximation of the approximate corrector for fixed $(s, p, R) \in \bar{\Omega} \times \mathbb{R}^{n} \times \mathcal{S}^{n \times n}$. For $\sigma \in(0,1)$ we consider the problem (3.5), i.e., the problem of finding a strong solution $v^{\sigma}=v^{\sigma}(\cdot ; s, p, R)$ to

$$
\sup _{\alpha \in \Lambda}\left\{-A_{s}^{\alpha}: D^{2} v^{\sigma}+\sigma v^{\sigma}-g_{s, p, R}^{\alpha}\right\}=0 \quad \text { in } \mathbb{R}^{n}, \quad v^{\sigma}(\cdot ; s, p, R) \in H_{\mathrm{per}}^{2}(Y) .
$$

Recall the notation $A_{s}^{\alpha}(y):=A_{s}(y, \alpha):=A(s, y, \alpha)$ and

$$
g_{s, p, R}^{\alpha}(y):=g_{s, p, R}(y, \alpha):=A^{\alpha}(s, y): R+b^{\alpha}(s, y) \cdot p+f^{\alpha}(s, y)
$$

for $y \in \mathbb{R}^{n}$ and $\alpha \in \Lambda$ from (3.3).

Note that $g_{s, p, R}: \mathbb{R}^{n} \times \Lambda \rightarrow \mathbb{R}$ is continuous and that $g_{s, p, R}^{\alpha}$ is $Y$-periodic for fixed $\alpha \in \Lambda$ and Lipschitz on $\mathbb{R}^{n}$ uniformly in $\alpha$. We also note that we have the Cordes condition (3.2), which yields

$$
\left|A_{s}\right|^{2}+\frac{\sigma^{2}}{\lambda_{\sigma}^{2}} \leq \frac{1}{n+\delta}\left(\operatorname{tr}\left(A_{s}\right)+\frac{\sigma}{\lambda_{\sigma}}\right)^{2} \quad \text { in } \mathbb{R}^{n} \times \Lambda,
$$

where $\lambda_{\sigma}>0$ is given by

$$
\lambda_{\sigma}:=\sigma \lambda
$$


The corresponding scaling function $\gamma^{\alpha}(y):=\gamma(y, \alpha)$ is defined by (compare with (2.4))

$$
\gamma:=\left(\left|A_{s}\right|^{2}+\frac{\sigma^{2}}{\lambda_{\sigma}^{2}}\right)^{-1}\left(\operatorname{tr}\left(A_{s}\right)+\frac{\sigma}{\lambda_{\sigma}}\right) .
$$

Observe that (3.7) is the Cordes condition (2.3) for the problem (3.6) with Cordes constants $\delta$ and $\lambda_{\sigma}$. Therefore, Theorem 2.1 ensures the well-posedness of the problem (3.6), i.e., the existence and uniqueness of a strong $Y$-periodic solution. We apply the mixed finite element method from section 2.4 to problem (3.6) to obtain an approximation.

The scheme from section 2 applied to the problem (3.6) yields an approximation $\left(m_{h}^{\sigma},\left(w_{h}^{\sigma}, v_{h}^{\sigma}\right)\right) \in M_{h} \times X_{h}$, whose existence and uniqueness are guaranteed by Theorem 2.7, satisfying the error bound

$$
\left\|\left(\nabla v^{\sigma}-w_{h}^{\sigma}, v^{\sigma}-v_{h}^{\sigma}\right)\right\|_{\lambda_{\sigma}} \leq C_{e}\left(\delta, \lambda_{\sigma}, n\right) \inf _{\left(w_{h}^{\prime}, u_{h}^{\prime}\right) \in X_{h}}\left\|\left(\nabla v^{\sigma}-w_{h}^{\prime}, v^{\sigma}-u_{h}^{\prime}\right)\right\|_{\lambda_{\sigma}},
$$

and we have that $C_{e}\left(\delta, \lambda_{\sigma}, n\right) \leq C_{e}(\delta, \lambda, n)$ for all $\sigma \in(0,1)$. In particular, in view of Remark 3.6, we have the boundedness of the sequence of numerical approximations in the sense that $\left\|\left(w_{h}^{\sigma}, v_{h}^{\sigma}\right)\right\|_{\lambda_{\sigma}} \leq C(\delta, \lambda, n) \sup _{\sigma \in(0,1)}\left\|\left(\nabla v^{\sigma}, v^{\sigma}\right)\right\|_{\lambda_{\sigma}}$, uniformly with respect to $h$ and $\sigma$.

For a shape-regular triangulation $\mathcal{T}_{h}$ on $Y$, denoting the Lagrange finite element space of degree $q \in \mathbb{N}$ over the triangulation by $\mathcal{S}^{q}\left(\mathcal{T}_{h}\right)$, we obtain the following approximation result.

Theorem 3.9 (error bound for the approximate corrector). For $\sigma \in(0,1)$, if we have $v^{\sigma}=v^{\sigma}(\cdot ; s, p, R) \in H^{2+r}(Y)$ for some $r \geq 0$ and the choice

$$
X_{h}:=\left(\mathcal{S}^{q}\left(\mathcal{T}_{h} ; \mathbb{R}^{n}\right) \cap W_{\text {per }}\left(Y ; \mathbb{R}^{n}\right)\right) \times\left(\mathcal{S}^{l}\left(\mathcal{T}_{h}\right) \cap H_{\text {per }}^{1}(Y)\right)
$$

for some $q, l \in \mathbb{N}$ and a shape-regular triangulation $\mathcal{T}_{h}$ on $Y$ (consistent with the requirement of periodicity), we find that

$$
\left\|\left(\nabla v^{\sigma}-w_{h}^{\sigma}, v^{\sigma}-v_{h}^{\sigma}\right)\right\|_{\lambda_{\sigma}} \leq C h^{\min \{r, q, l\}}\left\|\nabla v^{\sigma}\right\|_{H^{1+r}(Y)}
$$

for $h>0$ sufficiently small, with the constant $C>0$ only depending on $\delta, \lambda, n$ and interpolation constants.

Remark 3.10. The proof yields that the error constant can be taken to be

$$
C:=C_{e}(\delta, \lambda, n) C_{i}(1+\lambda)
$$

where $C_{i}$ is a constant arising from interpolation inequalities.

3.4. Approximation of the effective Hamiltonian. The approximation of the approximate corrector from the previous section allows us to obtain an approximation to the effective Hamiltonian as follows.

First, we note that with $\tilde{\alpha} \in(0,1)$ from Remark 3.6 we have that, for any $r \in$ $[0, \tilde{\alpha})$, there holds

$$
\sup _{\sigma \in(0,1)}\left\|\nabla v^{\sigma}(\cdot ; s, p, R)\right\|_{H^{1+r}(Y)} \lesssim \sup _{\sigma \in(0,1)}\left\|\nabla v^{\sigma}(\cdot ; s, p, R)\right\|_{C^{1, \tilde{\alpha}}\left(\mathbb{R}^{n}\right)} \lesssim 1+|p|+|R|,
$$

uniformly in $\sigma$. Using the error bound from Theorem 3.9, we deduce that

$$
\left\|\left(\nabla v^{\sigma}-w_{h}^{\sigma}, v^{\sigma}-v_{h}^{\sigma}\right)\right\|_{\lambda_{\sigma}} \lesssim h^{\min \{r, q, l\}}\left\|\nabla v^{\sigma}\right\|_{H^{1+r}(Y)} \lesssim h^{\min \{r, q, l\}}(1+|p|+|R|)
$$


with a constant independent of $\sigma$ and the choice of $(s, p, R)$. In particular, by the definition of $\|\cdot \mid\|_{\lambda_{\sigma}}$, we have

$$
\left\|\sigma v^{\sigma}-\sigma v_{h}^{\sigma}\right\|_{L^{2}(Y)} \lesssim h^{\min \{r, q, l\}}(1+|p|+|R|) .
$$

We then define the approximated effective Hamiltonian as

$$
H_{\sigma, h}: \bar{\Omega} \times \mathbb{R}^{n} \times \mathcal{S}^{n \times n} \rightarrow \mathbb{R}, \quad H_{\sigma, h}(s, p, R):=-\sigma \int_{Y} v_{h}^{\sigma}(\cdot ; s, p, R) .
$$

Then, the following approximation result holds.

TheOREm 3.11 (approximation of the effective Hamiltonian). Let $\sigma \in(0,1)$ and $\left(w_{h}^{\sigma}, v_{h}^{\sigma}\right) \in X_{h}$ as in Theorem 3.9. Further let $H_{\sigma, h}$ be defined as in (3.10). Then, for $(s, p, R) \in \bar{\Omega} \times \mathbb{R}^{n} \times \mathcal{S}^{n \times n}$, we have the error bound

$$
\left|H_{\sigma, h}(s, p, R)-H(s, p, R)\right| \lesssim\left(h^{r}+\sigma\right)(1+|p|+|R|)
$$

for any $r \in[0, \tilde{\alpha})$ with $\tilde{\alpha} \in(0,1)$ from Remark 3.6 and $\sigma, h>0$ sufficiently small. More generally, for fixed $(s, p, R) \in \bar{\Omega} \times \mathbb{R}^{n} \times \mathcal{S}^{n \times n}$, we have

$$
\left|H_{\sigma, h}(s, p, R)-H(s, p, R)\right|=\mathcal{O}\left(h^{\min \{r, q, l\}}+\sigma\right)
$$

for any $r \geq 0$ such that $\left\{\left\|\nabla v^{\sigma}(\cdot ; s, p, R)\right\|_{H^{1+r}(Y)}\right\}_{\sigma \in(0,1)}$ is uniformly bounded.

Whereas the effective Hamiltonian $H$ is a convex function in the variable $R \in$ $\mathcal{S}^{n \times n}$, it does not automatically follow that this property is inherited by the approximated effective Hamiltonian $H_{\sigma, h}$. Notwithstanding this, the numerical experiments performed in the next section make no use of the convexity (or otherwise) of $H_{\sigma, h}$.

\section{Numerical experiments.}

4.1. Set-up. We consider the problem of approximating the solution $u_{\varepsilon}$ to the HJB equation

$$
\left\{\begin{aligned}
\sup _{\alpha \in \Lambda}\left\{-A^{\alpha}\left(\frac{\cdot}{\varepsilon}\right): D^{2} u_{\varepsilon}+u_{\varepsilon}-1\right\} & =0 & & \text { in } \Omega, \\
u_{\varepsilon} & =0 & & \text { on } \partial \Omega,
\end{aligned}\right.
$$

where $\Omega:=(0,1)^{2} \subseteq \mathbb{R}^{2}$ is the unit square and $\Lambda:=[0,1]$. The coefficient $A$ has the structure

$$
A: \mathbb{R}^{2} \times \Lambda \rightarrow \mathcal{S}^{2 \times 2}, \quad A(y, \alpha):=A^{\alpha}(y):=\left(a_{0}(y)+\alpha a_{1}(y)\right) B
$$

for $Y$-periodic functions $a_{0}, a_{1}: \mathbb{R}^{2} \rightarrow(0, \infty)$ and a symmetric positive definite matrix $B \in \mathcal{S}^{2 \times 2}$. The homogenized problem (3.4) is then given by

$$
\left\{\begin{array}{rlrl}
u_{0}+H\left(D^{2} u_{0}\right) & =0 & & \text { in } \Omega, \\
u_{0}=0 & & \text { on } \partial \Omega,
\end{array}\right.
$$

and an explicit expression for the effective Hamiltonian, according to [18, section 2.2], is given by

$H: \mathcal{S}^{2 \times 2} \rightarrow \mathbb{R}, \quad H(R)=\max \left\{-\left(\int_{Y} \frac{1}{a_{0}}\right)^{-1} B: R,-\left(\int_{Y} \frac{1}{a_{0}+a_{1}}\right)^{-1} B: R\right\}-1$. 
Explicitly, we choose in our numerical experiments

$$
B:=\left(\begin{array}{cc}
2 & -1 \\
-1 & 4
\end{array}\right), \quad a_{0} \equiv 1, \quad a_{1}\left(y_{1}, y_{2}\right):=\sin ^{2}\left(2 \pi y_{1}\right) \cos ^{2}\left(2 \pi y_{2}\right)+1 .
$$

4.2. First experiment: Approximation of the effective Hamiltonian at a point. Our objective in the first numerical experiment is to investigate the approximation of the effective Hamiltonian $H(R)$ by the numerically computed approximate Hamiltonian $H_{\sigma, h}(R)$ at some given point $R \in \mathcal{S}^{2 \times 2}$. We choose

$$
R:=\left(\begin{array}{cc}
-2 & 1 \\
1 & -3
\end{array}\right)
$$

as a negative definite matrix so that the maximum in (4.1) is realized by the term involving the harmonic integral mean of $a_{0}+a_{1}$ (i.e., the term involving $\left.\left[\int_{Y}\left(a_{0}+a_{1}\right)^{-1}\right]^{-1}\right)$. For our discretization, we choose a continuous piecewise affine discretization with $q=l=1$ and $M_{h}:=\{0\}$. In order to compare the experimental results with the theoretical bound of Theorem 3.11, we consider the convergence in $h$ and $\sigma$ separately. We test convergence with respect to $h$ by fixing a (sufficiently small) value $\sigma=0.01$ and choosing a uniform mesh-refinement of the periodicity cell $Y=(0,1)^{2}$. Since the error bound for the approximate corrector from Theorem 3.9 is given in the norm $\|\cdot\|_{\lambda_{\sigma}}$, we first numerically test the convergence rate predicted by Theorem 3.9. The exact approximate corrector $v^{\sigma}$ is unknown, and thus we instead compute the a posteriori error estimator

$$
\eta(h):=\left\|F_{\gamma}\left[\left(w_{h}, u_{h}\right)\right]\right\|_{L^{2}(Y)}^{2}+\sigma_{1}\left\|\operatorname{rot}\left(w_{h}\right)\right\|_{L^{2}(Y)}^{2}+\sigma_{2}\left\|w_{h}-\nabla u_{h}\right\|_{L^{2}(Y)}^{2},
$$

which is, up to a constant factor, equivalent to the error in Theorem 3.9; see Theorem 2.9 and Remark 2.10. The convergence histories of $\eta / 100$ and the relative error

$$
\frac{\left|H_{\sigma, h}(R)-H(R)\right|}{|H(R)|}
$$

are displayed in Figure 1. As we are mainly interested in the rate of convergence, we plot $\eta / 100$ so that both error quantities can be shown in the same diagram.

As expected from Theorem 3.9, the error estimator is of order $h$, whereas we observe cubic convergence with respect to $h$ for the relative error of the effective Hamiltonian at the point $R$. This rate is higher than predicted by Theorem 3.11, which is based on an error estimate in the norm $\|\cdot \cdot\|_{\lambda_{\sigma}}$ and is therefore indeed expected to overestimate the actual error between $H_{\sigma, h}(R)$ and $H(R)$ related to the weaker integral functional from (3.10).

Next, we test convergence with respect to $\sigma$ by fixing a fine mesh size $h=\sqrt{2} \times 2^{-7}$ and letting $\sigma$ vary from $2^{4}$ to $2^{-7}$. The convergence history of the relative error is displayed in Figure 2.

We observe linear convergence with respect to $\sigma$, which indicates that the bound in Theorem 3.11 is sharp in $\sigma$.

4.3. Second experiment: Numerical approximation of the homogenized problem. The second numerical experiment is devoted to the approximation of the effective problem (3.4). We first note that the discretization on the scales $\Omega$ and $Y$ leads to a two-scale approach. We denote the triangulation of $\Omega$ by $\mathcal{T}_{h}^{\Omega}$ with mesh size $h_{\Omega}$ and the triangulation of $Y$ by $\mathcal{T}_{h}^{Y}$ with mesh size $h_{Y}$. In view of the regularity 


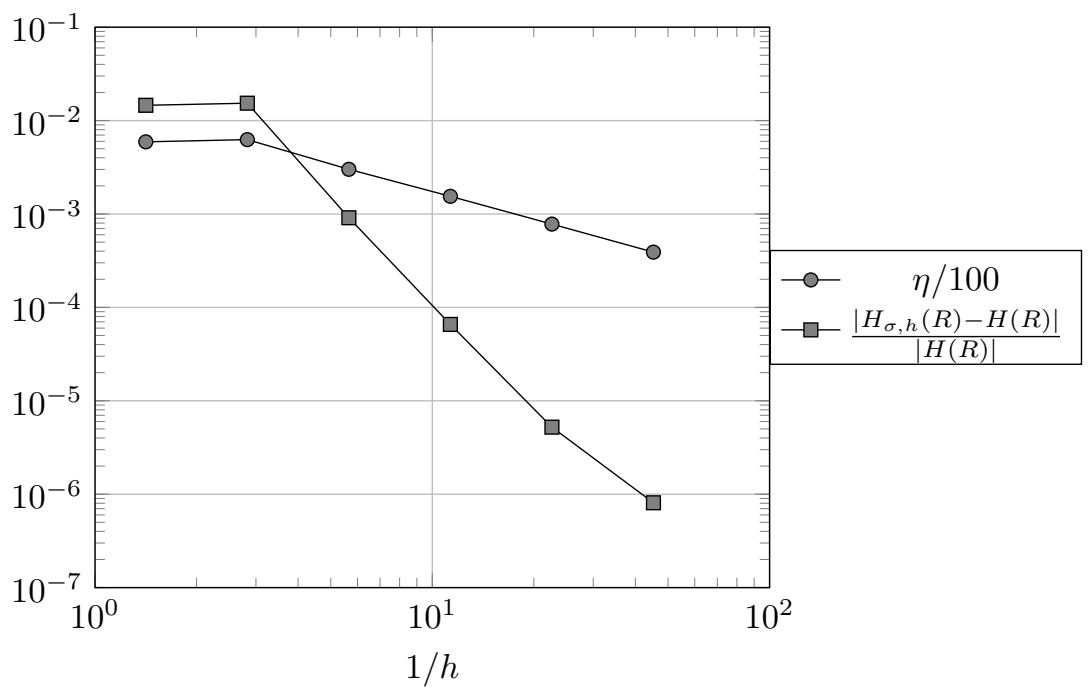

FIG. 1. Error estimator and approximation error between $H(R)$ by $H_{\sigma, h}(R)$ under mesh refinement with fixed $\sigma=0.01$.

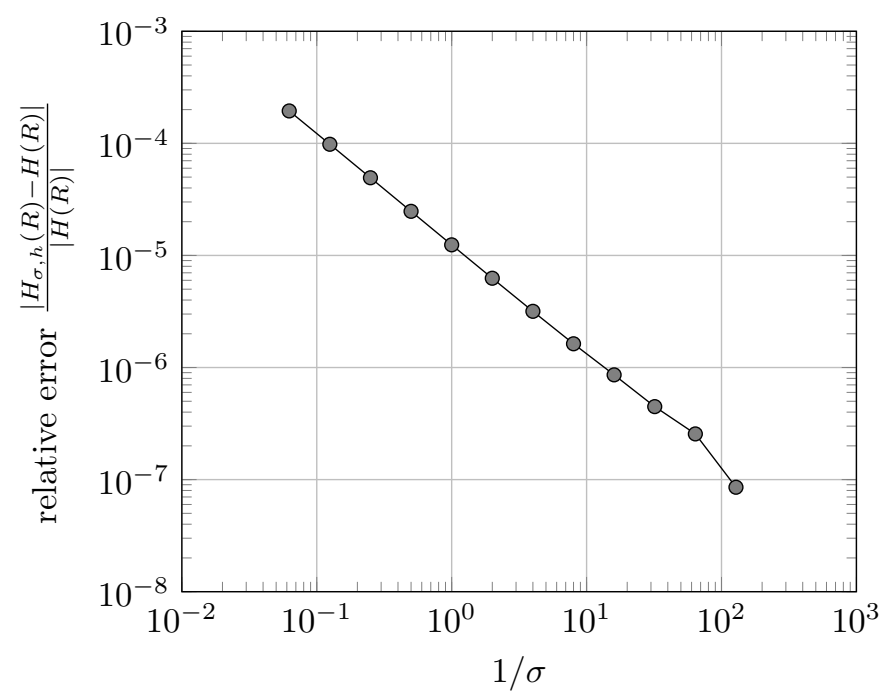

Fig. 2. Approximation of $H(R)$ by $H_{\sigma, h}(R)$ for varying $\sigma$ with fixed mesh size $h=\sqrt{2} \times 2^{-7}$.

result from Remark 3.8, we discretize the solution $u_{0}$ of this fully nonlinear equation by a least-squares approach, which is explained in the following. We discretize functions over $\Omega$ using the finite element space consisting of continuous piecewise affine functions

$$
\mathcal{S}_{0}^{1}\left(\mathcal{T}_{h}^{\Omega}\right)
$$

satisfying a homogeneous Dirichlet boundary condition, and their gradients by vectorvalued continuous piecewise affine finite elements

$$
\mathcal{S}^{1}\left(\mathcal{T}_{h}^{\Omega} ; \mathbb{R}^{2}\right)
$$




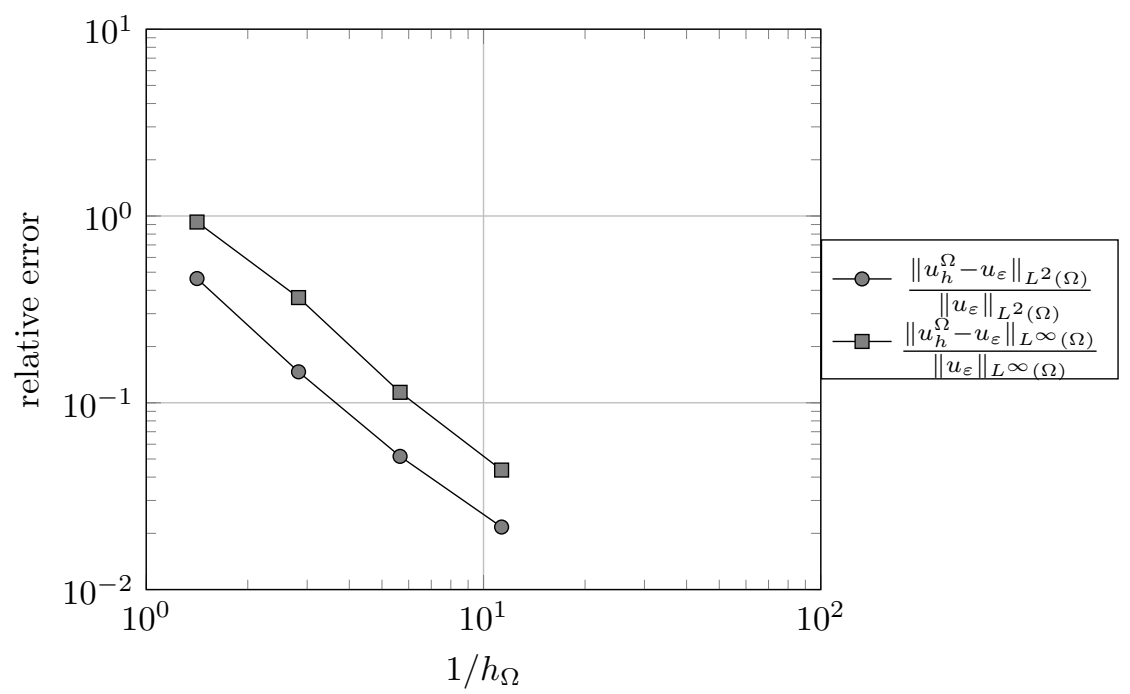

FIG. 3. Convergence history under mesh refinement of $\Omega$ for the approximation of the solution $u_{0}$ to the effective equation. The reference solution $u_{\varepsilon}$ is computed for $\varepsilon=0.1$. The cell problem is solved with $h_{Y}=\sqrt{2} \times 2^{-2}$ and $\sigma=0.1$.

Given $w_{h}^{\Omega} \in \mathcal{S}^{1}\left(\mathcal{T}_{h}^{\Omega} ; \mathbb{R}^{2}\right)$, we say that $D w_{h}^{\Omega}$ is the discrete Hessian of some $u_{h}^{\Omega} \in$ $\mathcal{S}_{0}^{1}\left(\mathcal{T}_{h}^{\Omega}\right)$ if it satisfies

$$
\int_{\Omega} w_{h}^{\Omega} \cdot v=\int_{\Omega} \nabla u_{h}^{\Omega} \cdot v \quad \forall v \in \mathcal{S}^{1}\left(\mathcal{T}_{h}^{\Omega} ; \mathbb{R}^{2}\right)
$$

and we write $D_{h}^{2} u_{h}^{\Omega}:=D w_{h}^{\Omega}$. The discrete Hessian $D_{h}^{2} u_{h}^{\Omega}$ is expected to be discontinuous across the element boundaries. In order to define a function that represents the evaluation of the discretized approximate Hamiltonian $H_{\sigma, h_{Y}}$ at $D_{h}^{2} u_{h}^{\Omega}$, we define the continuous and piecewise affine function $\tilde{H}_{\sigma, h_{Y}}\left(D^{2} u_{h}^{\Omega}\right)$ by nodal averaging of the piecewise constant function

$$
x \mapsto H_{\sigma, h_{Y}}(\operatorname{mid}(T)) \quad \text { for } T \in \mathcal{T}_{h}^{\Omega} \text { with } x \in T
$$

(defined a.e. in $\Omega$ ), where $\operatorname{mid}(T)$ denotes the barycenter of $T$. We then define the numerical approximation $u_{h}^{\Omega}=u_{h}^{\Omega}\left(h_{\Omega}, \sigma, h_{Y}\right)$ as a minimizer of the following leastsquares functional:

$$
u_{h}^{\Omega} \in \underset{v_{h}^{\Omega} \in \mathcal{S}_{0}^{1}\left(\mathcal{T}_{h}^{\Omega}\right)}{\arg \min }\left\|v_{h}^{\Omega}+\tilde{H}_{\sigma, h_{Y}}\left(D_{h}^{2} v_{h}^{\Omega}\right)\right\|_{L^{2}(\Omega)}^{2} .
$$

In our implementation, we computed the minimizer by using the built-in function fmincon of MATLAB, without prescribing any derivative information because we are not aware of any (semi)smoothness properties of the solution operator. We choose $\sigma=0.1$ and $h_{Y}=\sqrt{2} \times 2^{-2}$ fixed and consider a sequence of uniformly refined triangulations of $\Omega$ with mesh sizes $h_{\Omega} \in \sqrt{2} \times 2^{-\{1,2,3,4\}}$. For the error computation, we use as a reference solution the approximation of $u_{\varepsilon}$ with $\varepsilon=0.1$ on a triangulation with mesh-size $\sqrt{2} \times 2^{-7}$. The convergence history of the errors in the $L^{\infty}$ and $L^{2}$ norms is displayed in Figure 3. 
For both error norms we observe a convergence order of $h_{\Omega}^{3 / 2}$, which indicates that the effective problem with the chosen data is possibly more regular than predicted in Remark 3.8.

\section{Collection of the proofs.}

\subsection{Proofs for section 2.}

Proof of Lemma 2.2. For the first part, we use successively properties for the supremum, the Cauchy-Schwarz inequality, simple calculation, and the Cordes condition (2.3) to obtain

$$
\begin{aligned}
\left|F_{\gamma}[(w, u)]-F_{\gamma}\left[\left(w^{\prime}, u^{\prime}\right)\right]-L_{\lambda}\left(w-w^{\prime}, u-u^{\prime}\right)\right|^{2} \\
\leq \sup _{\alpha \in \Lambda} \mid \gamma^{\alpha}\left(-A^{\alpha}: D\left(w-w^{\prime}\right)-b^{\alpha} \cdot \nabla\left(u-u^{\prime}\right)+c^{\alpha}\left(u-u^{\prime}\right)\right) \\
\quad+\nabla \cdot\left(w-w^{\prime}\right)-\left.\lambda\left(u-u^{\prime}\right)\right|^{2} \\
\leq \sup _{\alpha \in \Lambda}\left(\left|-\gamma^{\alpha} A^{\alpha}+I\right|^{2}+\frac{\left|\gamma^{\alpha} b^{\alpha}\right|^{2}}{2 \lambda}+\frac{\left|\gamma^{\alpha} c^{\alpha}-\lambda\right|^{2}}{\lambda^{2}}\right) \\
\quad \times\left(\left|D\left(w-w^{\prime}\right)\right|^{2}+2 \lambda\left|\nabla\left(u-u^{\prime}\right)\right|^{2}+\lambda^{2}\left|u-u^{\prime}\right|^{2}\right) \\
=\sup _{\alpha \in \Lambda}\left(n+1-\frac{\left(\operatorname{tr}\left(A^{\alpha}\right)+\frac{c^{\alpha}}{\lambda}\right)^{2}}{\left|A^{\alpha}\right|^{2}+\frac{\left|b^{\alpha}\right|^{2}}{2 \lambda}+\frac{\left|c^{\alpha}\right|^{2}}{\lambda^{2}}}\right) \\
\quad \times\left(\left|D\left(w-w^{\prime}\right)\right|^{2}+2 \lambda\left|\nabla\left(u-u^{\prime}\right)\right|^{2}+\lambda^{2}\left|u-u^{\prime}\right|^{2}\right) \\
\leq(1-\delta)\left(\left|D\left(w-w^{\prime}\right)\right|^{2}+2 \lambda\left|\nabla\left(u-u^{\prime}\right)\right|^{2}+\lambda^{2}\left|u-u^{\prime}\right|^{2}\right)
\end{aligned}
$$

almost everywhere in $Y$, which yields the estimate (2.11).

For the second part, we use (2.10), integration by parts, and Young's inequality to find

$$
\begin{aligned}
\|(w, u)\|_{\lambda}^{2} & =\|\operatorname{rot}(w)\|_{L^{2}(Y)}^{2}+\|\nabla \cdot w\|_{L^{2}(Y)}^{2}+2 \lambda\|\nabla u\|_{L^{2}(Y)}^{2}+\lambda^{2}\|u\|_{L^{2}(Y)}^{2} \\
& =\|\operatorname{rot}(w)\|_{L^{2}(Y)}^{2}+\|-\nabla \cdot w+\lambda u\|_{L^{2}(Y)}^{2}+2 \lambda \int_{Y}(\nabla u-w) \cdot \nabla u \\
& \leq\|\operatorname{rot}(w)\|_{L^{2}(Y)}^{2}+\left\|L_{\lambda}(w, u)\right\|_{L^{2}(Y)}^{2}+\frac{\lambda}{\rho}\|\nabla u-w\|_{L^{2}(Y)}^{2}+\lambda \rho\|\nabla u\|_{L^{2}(Y)}^{2} \\
& \leq\|\operatorname{rot}(w)\|_{L^{2}(Y)}^{2}+\left\|L_{\lambda}(w, u)\right\|_{L^{2}(Y)}^{2}+\frac{\lambda}{\rho}\|\nabla u-w\|_{L^{2}(Y)}^{2}+\frac{\rho}{2}\|(w, u)\|_{\lambda}^{2},
\end{aligned}
$$

which yields the Miranda-Talenti-type estimate (2.12).

Proof of Lemma 2.3. We are going to prove the claimed results (i), (ii), (iii) separately.

(i) By (2.11), Young's inequality, and the Miranda-Talenti-type estimate (2.12) with the choice $\rho=2-2 \sqrt{1-\delta}$, we find that

$$
\begin{aligned}
& \begin{array}{l}
a\left((w, u),\left(w-w^{\prime}, u-u^{\prime}\right)\right)-a\left(\left(w^{\prime}, u^{\prime}\right),\left(w-w^{\prime}, u-u^{\prime}\right)\right) \\
\quad-\sigma_{1}\left\|\operatorname{rot}\left(w-w^{\prime}\right)\right\|_{L^{2}(Y)}^{2}-\sigma_{2}\left\|\nabla\left(u-u^{\prime}\right)-\left(w-w^{\prime}\right)\right\|_{L^{2}(Y)}^{2}
\end{array} \\
& =\int_{Y}\left(F_{\gamma}[(w, u)]-F_{\gamma}\left[\left(w^{\prime}, u^{\prime}\right)\right]\right) L_{\lambda}\left(w-w^{\prime}, u-u^{\prime}\right) \\
& \geq\left\|L_{\lambda}\left(w-w^{\prime}, u-u^{\prime}\right)\right\|_{L^{2}(Y)}^{2}-\sqrt{1-\delta}\left\|\left(w-w^{\prime}, u-u^{\prime}\right)\right\|_{\lambda}\left\|L_{\lambda}\left(w-w^{\prime}, u-u^{\prime}\right)\right\|_{L^{2}(Y)}
\end{aligned}
$$




$$
\begin{gathered}
\geq \frac{2-\sqrt{1-\delta}}{2}\left\|L_{\lambda}\left(w-w^{\prime}, u-u^{\prime}\right)\right\|_{L^{2}(Y)}^{2}-\frac{\sqrt{1-\delta}}{2}\left\|\left(w-w^{\prime}, u-u^{\prime}\right)\right\|_{\lambda}^{2} \\
\geq \frac{1-\sqrt{1-\delta}}{2}\left\|L_{\lambda}\left(w-w^{\prime}, u-u^{\prime}\right)\right\|_{L^{2}(Y)}^{2}-\frac{1}{2}\left\|\operatorname{rot}\left(w-w^{\prime}\right)\right\|_{L^{2}(Y)}^{2} \\
-\frac{\lambda}{4-4 \sqrt{1-\delta}}\left\|\nabla\left(u-u^{\prime}\right)-\left(w-w^{\prime}\right)\right\|_{L^{2}(Y)}^{2} .
\end{gathered}
$$

Therefore, by the definition of the constants $\sigma_{1}, \sigma_{2}$ and the Miranda-Talenti-type estimate (2.12) with the choice $\rho=1$, we conclude that

$$
\begin{aligned}
& a\left((w, u),\left(w-w^{\prime}, u-u^{\prime}\right)\right)-a\left(\left(w^{\prime}, u^{\prime}\right),\left(w-w^{\prime}, u-u^{\prime}\right)\right) \\
& \geq \frac{1-\sqrt{1-\delta}}{2}\left(\left\|L_{\lambda}\left(w-w^{\prime}, u-u^{\prime}\right)\right\|_{L^{2}(Y)}^{2}+\left\|\operatorname{rot}\left(w-w^{\prime}\right)\right\|_{L^{2}(Y)}^{2}\right. \\
& \left.\quad+\lambda\left\|\nabla\left(u-u^{\prime}\right)-\left(w-w^{\prime}\right)\right\|_{L^{2}(Y)}^{2}\right)
\end{aligned}
$$

which is the claimed inequality.

(ii) We note that we have

$$
\left\|L_{\lambda}(w, u)\right\|_{L^{2}(Y)} \leq \sqrt{2}\|(w, u)\|_{\lambda} \quad \forall(w, u) \in X,
$$

as there holds $\|\nabla \cdot w\|_{L^{2}(Y)} \leq\|D w\|_{L^{2}(Y)}$ for any $w \in W_{\text {per }}\left(Y ; \mathbb{R}^{n}\right)$ by $(2.10)$. We bound the terms arising in the quantity on the left-hand side of (2.13) separately. For the term involving the nonlinearity, using (2.11), we have

$$
\begin{aligned}
& \left|\int_{Y}\left(F_{\gamma}[(w, u)]-F_{\gamma}\left[\left(w^{\prime}, u^{\prime}\right)\right]\right) L_{\lambda}(z, v)\right| \\
& \leq\left\|L_{\lambda}(z, v)\right\|_{L^{2}(Y)}\left(\left\|L_{\lambda}\left(w-w^{\prime}, u-u^{\prime}\right)\right\|_{L^{2}(Y)}+\sqrt{1-\delta}\left\|\left(w-w^{\prime}, u-u^{\prime}\right)\right\|_{\lambda}\right) \\
& \leq \sqrt{2}(\sqrt{2}+\sqrt{1-\delta})\left\|\left(w-w^{\prime}, u-u^{\prime}\right)\right\|_{\lambda}\|(z, v)\|_{\lambda} .
\end{aligned}
$$

For the term multiplying the constant $\sigma_{1}$, we have

$$
\begin{aligned}
\left|\sigma_{1} \int_{Y} \operatorname{rot}\left(w-w^{\prime}\right) \cdot \operatorname{rot}(z)\right| & \leq \sigma_{1}\left\|D\left(w-w^{\prime}\right)\right\|_{L^{2}(Y)}\|D z\|_{L^{2}(Y)} \\
& \leq \sigma_{1}\left\|\left(w-w^{\prime}, u-u^{\prime}\right)\right\|\left\|_{\lambda}\right\|(z, v) \|_{\lambda}
\end{aligned}
$$

as there holds $\|\operatorname{rot}(w)\|_{L^{2}(Y)} \leq\|D w\|_{L^{2}(Y)}$ for any $w \in W_{\text {per }}\left(Y ; \mathbb{R}^{n}\right)$ by $(2.10)$. For the term multiplying the constant $\sigma_{2}$, we have by the triangle, Poincaré (2.8), and Cauchy-Schwarz inequalities that

$$
\begin{aligned}
& \left|\sigma_{2} \int_{Y}\left(\nabla\left(u-u^{\prime}\right)-\left(w-w^{\prime}\right)\right) \cdot(\nabla v-z)\right| \\
& \leq \sigma_{2}\left(\left\|\nabla\left(u-u^{\prime}\right)\right\|_{L^{2}(Y)}+\frac{\sqrt{n}}{\pi}\left\|D\left(w-w^{\prime}\right)\right\|_{L^{2}(Y)}\right)\left(\|\nabla v\|_{L^{2}(Y)}+\frac{\sqrt{n}}{\pi}\|D z\|_{L^{2}(Y)}\right) \\
& \leq \sigma_{2}\left(\frac{1}{2 \lambda}+\frac{n}{\pi^{2}}\right)\left\|\left(w-w^{\prime}, u-u^{\prime}\right)\right\|\left\|_{\lambda}\right\|(z, v) \|_{\lambda} .
\end{aligned}
$$


Altogether, we obtain the claimed inequality (2.13) with the constant

$$
C_{L}=2+\sqrt{2} \sqrt{1-\delta}+\sigma_{1}+\sigma_{2}\left(\frac{1}{2 \lambda}+\frac{n}{\pi^{2}}\right)
$$

which is identical to the one given in Lemma 2.3(ii) using that $\sigma_{2}=\lambda \tilde{\sigma}_{2}$.

(iii) For any $m^{\prime} \in M \backslash\{0\}$ we have $\left(0, m^{\prime}\right) \in X$ and hence

$$
\begin{aligned}
\sup _{\left(w^{\prime}, u^{\prime}\right) \in X \backslash\{0\}} \frac{b\left(m^{\prime},\left(w^{\prime}, u^{\prime}\right)\right)}{\left\|\left(w^{\prime}, u^{\prime}\right)\right\|_{\lambda}} & \geq \frac{b\left(m^{\prime},\left(0, m^{\prime}\right)\right)}{\left\|\left(0, m^{\prime}\right)\right\|_{\lambda}} \\
& =\frac{\left\|\nabla m^{\prime}\right\|_{L^{2}(Y)}^{2}}{\sqrt{2 \lambda\left\|\nabla m^{\prime}\right\|_{L^{2}(Y)}^{2}+\lambda^{2}\left\|m^{\prime}\right\|_{L^{2}(Y)}^{2}}} \\
& \geq \frac{\left\|\nabla m^{\prime}\right\|_{L^{2}(Y)}}{\sqrt{2 \lambda+\frac{n}{\pi^{2}} \lambda^{2}}}
\end{aligned}
$$

by Poincaré's inequality (2.7) (recall that $M \subset W_{\text {per }}(Y)$ ), which yields the claimed result (2.14).

Proof of Theorem 2.5. The existence of a unique solution $(m,(w, u)) \in M \times X$ to (2.9) follows from the Brezzi-splitting; see [5] and [21, Proposition 2.5], as we have the monotonicity and Lipschitz continuity for $a$ and an inf-sup condition from Lemma 2.3. For the second part of the claim, i.e., that $m=0, u \in H_{\mathrm{per}}^{2}(Y)$ with $w=\nabla u$ and $u$ is the solution to (2.1), we note that $L_{\lambda}$ is surjective from the set $X_{g}:=\left\{\left(w^{\prime}, u^{\prime}\right) \in X: w^{\prime}=\nabla u^{\prime}\right\}$ onto $L^{2}(Y)$. We first test the mixed formulation (2.9) with pairs $\left(w^{\prime}, u^{\prime}\right)$ from $X_{g}$ to obtain $F_{\gamma}[(w, u)]=0$ almost everywhere and then with the solution pair $(w, u)$ to find that $w=\nabla u$ and thus $u \in H_{\mathrm{per}}^{2}(Y)$. We conclude the proof by noting that this implies that $u$ is the solution to (2.5) (and hence to (2.1) by Theorem 2.1) and that $m=0$.

Proof of Lemma 2.6. We use the triangle, Poincaré (2.8), and Cauchy-Schwarz inequalities to obtain that

$$
\begin{aligned}
b\left(m^{\prime},\left(w^{\prime}, u^{\prime}\right)\right) & \leq\left\|\nabla m^{\prime}\right\|_{L^{2}(Y)}\left(\left\|\nabla u^{\prime}\right\|_{L^{2}(Y)}+\frac{\sqrt{n}}{\pi}\left\|D w^{\prime}\right\|_{L^{2}(Y)}\right) \\
& \leq \sqrt{\frac{1}{2 \lambda}+\frac{n}{\pi^{2}}}\left\|\nabla m^{\prime}\right\|_{L^{2}(Y)}\left\|\left(w^{\prime}, u^{\prime}\right)\right\|_{\lambda}
\end{aligned}
$$

for all $\left(m^{\prime},\left(w^{\prime}, u^{\prime}\right)\right) \in M \times X$.

The discrete inf-sup condition holds, as for $m_{h}^{\prime} \in M_{h} \backslash\{0\}$ we have $\left(0, m_{h}^{\prime}\right) \in$ $X_{h} \backslash\{0\}$ since $M_{h} \subset U_{h} \cap M$, and hence

$$
\begin{aligned}
\sup _{\left(w_{h}^{\prime}, u_{h}^{\prime}\right) \in X_{h} \backslash\{0\}} \frac{b\left(m_{h}^{\prime},\left(w_{h}^{\prime}, u_{h}^{\prime}\right)\right)}{\left\|\left(w_{h}^{\prime}, u_{h}^{\prime}\right)\right\|_{\lambda}} & \geq \frac{b\left(m_{h}^{\prime},\left(0, m_{h}^{\prime}\right)\right)}{\left\|\left(0, m_{h}^{\prime}\right)\right\|_{\lambda}} \\
& =\frac{\left\|\nabla m_{h}^{\prime}\right\|_{L^{2}(Y)}^{2}}{\sqrt{2 \lambda\left\|\nabla m_{h}^{\prime}\right\|_{L^{2}(Y)}^{2}+\lambda^{2}\left\|m_{h}^{\prime}\right\|_{L^{2}(Y)}^{2}}} \\
& \geq \frac{\left\|\nabla m_{h}^{\prime}\right\|_{L^{2}(Y)}}{\sqrt{2 \lambda+\frac{n}{\pi^{2}} \lambda^{2}}}
\end{aligned}
$$

by Poincaré's inequality (2.7) (recall that $M \subset W_{\text {per }}(Y)$ ), which yields the claimed result (2.16). 
Proof of Theorem 2.7. We only show the error bound, as the existence and uniqueness of solutions for (2.15) follows from Lemmas 2.3 and 2.6 in a standard way; see [21, Proposition 3.1].

Step 1. We introduce the discrete kernel

$$
Z_{h}:=\left\{\left(w_{h}^{\prime}, u_{h}^{\prime}\right) \in X_{h}: b\left(m_{h}^{\prime},\left(w_{h}^{\prime}, u_{h}^{\prime}\right)\right)=0 \quad \forall m_{h}^{\prime} \in M_{h}\right\}
$$

and claim that there holds

$$
\left\|\left(w-w_{h}, u-u_{h}\right)\right\|_{\lambda} \leq \frac{C_{L}}{C_{M}} \inf _{\left(w_{h}^{\prime}, u_{h}^{\prime}\right) \in Z_{h}}\left\|\left(w-w_{h}^{\prime}, u-u_{h}^{\prime}\right)\right\|_{\lambda} .
$$

Indeed, we use successively the monotonicity from Lemma 2.3(i), the solution property of $(w, u)$ from Theorem 2.5, and the fact that $\left(w_{h}, u_{h}\right)$ solves the discrete problem (2.15), and the Lipschitz estimate from Lemma 2.3(ii) to find that

$$
\begin{aligned}
C_{M} & \left\|\left(w-w_{h}, u-u_{h}\right)\right\|_{\lambda}^{2} \\
& \leq a\left((w, u),\left(w-w_{h}, u-u_{h}\right)\right)-a\left(\left(w_{h}, u_{h}\right),\left(w-w_{h}, u-u_{h}\right)\right) \\
& =-a\left(\left(w_{h}, u_{h}\right),(w, u)\right) \\
& =-a\left(\left(w_{h}, u_{h}\right),\left(w-w_{h}^{\prime}, u-u_{h}^{\prime}\right)\right) \\
& =a\left((w, u),\left(w-w_{h}^{\prime}, u-u_{h}^{\prime}\right)\right)-a\left(\left(w_{h}, u_{h}\right),\left(w-w_{h}^{\prime}, u-u_{h}^{\prime}\right)\right) \\
& \leq C_{L}\left\|\left(w-w_{h}, u-u_{h}\right)\right\|_{\lambda}\left\|\left(w-w_{h}^{\prime}, u-u_{h}^{\prime}\right)\right\|_{\lambda}
\end{aligned}
$$

for any $\left(w_{h}^{\prime}, u_{h}^{\prime}\right) \in Z_{h}$, which implies the desired estimate (5.2).

Step 2 . We let $\left(w_{*}, u_{*}\right) \in X_{h}$ denote the best-approximation to $(w, u)$ from $Z_{h}$, i.e.,

$$
\left\|\left(w-w_{*}, u-u_{*}\right)\right\|_{\lambda}=\inf _{\left(w_{h}^{\prime}, u_{h}^{\prime}\right) \in Z_{h}}\left\|\left(w-w_{h}^{\prime}, u-u_{h}^{\prime}\right)\right\|_{\lambda}
$$

and we derive a linear mixed problem for $\left(w_{*}, u_{*}\right)$.

By the discrete inf-sup condition (2.16), there exists $m_{*} \in M_{h}$ such that

$$
\left\{\begin{array}{rlrl}
\left\langle\left(w_{*}, u_{*}\right),\left(w_{h}^{\prime}, u_{h}^{\prime}\right)\right\rangle_{\lambda}+b\left(m_{*},\left(w_{h}^{\prime}, u_{h}^{\prime}\right)\right) & =\left\langle(w, u),\left(w_{h}^{\prime}, u_{h}^{\prime}\right)\right\rangle_{\lambda} & & \forall\left(w_{h}^{\prime}, u_{h}^{\prime}\right) \in X_{h}, \\
b\left(m_{h}^{\prime},\left(w_{*}, u_{*}\right)\right)=0 & & \forall m_{h}^{\prime} \in M_{h},
\end{array}\right.
$$

where $\langle\cdot, \cdot\rangle_{\lambda}: X \times X \rightarrow \mathbb{R}$ is the inner product given by

$$
\left\langle\left(w^{\prime}, u^{\prime}\right),\left(w^{\prime \prime}, u^{\prime \prime}\right)\right\rangle_{\lambda}:=\int_{Y} D w^{\prime}: D w^{\prime \prime}+2 \lambda \int_{Y} \nabla u^{\prime} \cdot \nabla u^{\prime \prime}+\lambda^{2} \int_{Y} u^{\prime} u^{\prime \prime} .
$$

We also note that the solution pair $(m,(w, u))$ satisfies the similar system (recall that $m=0$ )

$$
\left\{\begin{aligned}
\left\langle(w, u),\left(w^{\prime}, u^{\prime}\right)\right\rangle_{\lambda}+b\left(m,\left(w^{\prime}, u^{\prime}\right)\right) & =\left\langle(w, u),\left(w^{\prime}, u^{\prime}\right)\right\rangle_{\lambda} & & \forall\left(w^{\prime}, u^{\prime}\right) \in X, \\
b\left(m^{\prime},(w, u)\right) & =0 & & \forall m^{\prime} \in M .
\end{aligned}\right.
$$

Step 3 . We derive an error bound for $\left(w-w_{*}, u-u_{*}\right)$ in the $\|\cdot \cdot\|_{\lambda}$ norm using classical linear mixed finite element theory. 
Note that for any $\left(w^{\prime}, u^{\prime}\right),\left(w^{\prime \prime}, u^{\prime \prime}\right) \in X$, we have

$$
\left|\left\langle\left(w^{\prime}, u^{\prime}\right),\left(w^{\prime \prime}, u^{\prime \prime}\right)\right\rangle_{\lambda}\right| \leq\|\|\left(w^{\prime}, u^{\prime}\right)\left\|_{\lambda}\right\|\left(w^{\prime \prime}, u^{\prime \prime}\right)\left\|_{\lambda}, \quad\left\langle\left(w^{\prime}, u^{\prime}\right),\left(w^{\prime}, u^{\prime}\right)\right\rangle_{\lambda}=\right\|\left(w^{\prime}, u^{\prime}\right) \|_{\lambda}^{2} .
$$

In particular, we have boundedness and coercivity on the whole space, i.e.,

$$
\begin{aligned}
\left|\left\langle\left(w^{\prime}, u^{\prime}\right),\left(w^{\prime \prime}, u^{\prime \prime}\right)\right\rangle_{\lambda}\right| & \leq C_{a}\left\|\left(w^{\prime}, u^{\prime}\right)\right\|_{\lambda}\left\|\left(w^{\prime \prime}, u^{\prime \prime}\right)\right\|_{\lambda}, \\
\left\langle\left(w^{\prime}, u^{\prime}\right),\left(w^{\prime}, u^{\prime}\right)\right\rangle_{\lambda} & \geq c_{a}\left\|\left(w^{\prime}, u^{\prime}\right)\right\|_{\lambda}^{2}
\end{aligned}
$$

for all $\left(w^{\prime}, u^{\prime}\right),\left(w^{\prime \prime}, u^{\prime \prime}\right) \in X$ with the constants $C_{a}:=c_{a}:=1$. Further, from Lemma 2.6 we have the discrete inf-sup condition (2.16) with the constant $c_{b}$ and boundedness of $b$ with the constant $C_{b}$. Then, by linear mixed finite element theory (see [40]), we obtain

$$
\begin{aligned}
\left\|\left(w-w_{*}, u-u_{*}\right)\right\|_{\lambda} \leq & \left(1+\frac{C_{a}}{c_{a}}\right)\left(1+\frac{C_{b}}{c_{b}}\right) \inf _{\left(w_{h}^{\prime}, u_{h}^{\prime}\right) \in X_{h}}\left\|\left(w-w_{h}^{\prime}, u-u_{h}^{\prime}\right)\right\|_{\lambda} \\
& +\frac{C_{b}}{c_{a}} \inf _{m_{h}^{\prime} \in M_{h}}\left\|\nabla\left(m-m_{h}^{\prime}\right)\right\|_{L^{2}(Y)} \\
= & 2\left(1+\frac{C_{b}}{c_{b}}\right) \inf _{\left(w_{h}^{\prime}, u_{h}^{\prime}\right) \in X_{h}}\left\|\left(w-w_{h}^{\prime}, u-u_{h}^{\prime}\right)\right\|_{\lambda},
\end{aligned}
$$

where we have used that $m=0$ and $C_{a}=c_{a}=1$ in the last line.

Step 4. We conclude by combining (5.2), (5.3), and (5.4) to deduce that

$$
\begin{aligned}
\left\|\left(w-w_{h}, u-u_{h}\right)\right\|_{\lambda} & \leq \frac{C_{L}}{C_{M}}\left\|\left(w-w_{*}, u-u_{*}\right)\right\|_{\lambda} \\
& \leq 2 \frac{C_{L}}{C_{M}}\left(1+\frac{C_{b}}{c_{b}}\right)_{\left(w_{h}^{\prime}, u_{h}^{\prime}\right) \in X_{h}}\left\|\left(w-w_{h}^{\prime}, u-u_{h}^{\prime}\right)\right\|_{\lambda},
\end{aligned}
$$

which is the desired error bound.

Proof of Theorem 2.9. We use successively the monotonicity from Lemma 2.3(i), the solution property of $(w, u)$ from Theorem 2.5, the Cauchy-Schwarz inequality (note that $w=\nabla u$ ), the bound (5.1), and Young's inequality to show that

$$
\begin{aligned}
C_{M} \| & \left(w-w_{h}, u-u_{h}\right) \|_{\lambda}^{2} \\
\leq & a\left((w, u),\left(w-w_{h}, u-u_{h}\right)\right)-a\left(\left(w_{h}, u_{h}\right),\left(w-w_{h}, u-u_{h}\right)\right) \\
= & -a\left(\left(w_{h}, u_{h}\right),\left(w-w_{h}, u-u_{h}\right)\right) \\
\leq & \left\|F_{\gamma}\left[\left(w_{h}, u_{h}\right)\right]\right\|_{L^{2}(Y)}\left\|L_{\lambda}\left(w-w_{h}, u-u_{h}\right)\right\|_{L^{2}(Y)}+\sigma_{1}\left\|\operatorname{rot}\left(w_{h}\right)\right\|_{L^{2}(Y)}^{2} \\
& \quad+\sigma_{2}\left\|w_{h}-\nabla u_{h}\right\|_{L^{2}(Y)}^{2} \\
\leq & \sqrt{2}\left\|F_{\gamma}\left[\left(w_{h}, u_{h}\right)\right]\right\|_{L^{2}(Y)}\left\|\left(w-w_{h}, u-u_{h}\right)\right\|_{\lambda}+\sigma_{1}\left\|\operatorname{rot}\left(w_{h}\right)\right\|_{L^{2}(Y)}^{2} \\
\quad & \quad+\sigma_{2}\left\|w_{h}-\nabla u_{h}\right\|_{L^{2}(Y)}^{2} \\
\leq & C_{M}^{-1}\left\|F_{\gamma}\left[\left(w_{h}, u_{h}\right)\right]\right\|_{L^{2}(Y)}^{2}+\frac{C_{M}}{2}\left\|\left(w-w_{h}, u-u_{h}\right)\right\|_{\lambda}^{2}+\sigma_{1}\left\|\operatorname{rot}\left(w_{h}\right)\right\|_{L^{2}(Y)}^{2} \\
\quad & \quad+\sigma_{2}\left\|w_{h}-\nabla u_{h}\right\|_{L^{2}(Y)}^{2} \cdot
\end{aligned}
$$

Upon rearranging, we find the claimed a posteriori estimate. 
For the efficiency estimate, recall the solution property of $(w, u)$ from Theorem 2.5 , in particular $w=\nabla u$ and $F_{\gamma}[(w, u)]=0$ almost everywhere. With the Lipschitz property from Lemma 2.3(ii) and with Lemma 2.2, we then obtain

$$
\begin{aligned}
& C_{L}\left\|\left(w-w_{h}, u-u_{h}\right)\right\|_{\lambda}^{2}-\sigma_{1}\left\|\operatorname{rot}\left(w_{h}\right)\right\|_{L^{2}(Y)}^{2}-\sigma_{2}\left\|w_{h}-\nabla u_{h}\right\|_{L^{2}(Y)}^{2} \\
& \geq a\left((w, u),\left(w-w_{h}, u-u_{h}\right)\right)-a\left(\left(w_{h}, u_{h}\right),\left(w-w_{h}, u-u_{h}\right)\right)-\sigma_{1}\left\|\operatorname{rot}\left(w_{h}\right)\right\|_{L^{2}(Y)}^{2} \\
& \quad-\sigma_{2}\left\|w_{h}-\nabla u_{h}\right\|_{L^{2}(Y)}^{2} \\
&=-a\left(\left(w_{h}, u_{h}\right),\left(w-w_{h}, u-u_{h}\right)\right)-\sigma_{1}\left\|\operatorname{rot}\left(w_{h}\right)\right\|_{L^{2}(Y)}^{2}-\sigma_{2}\left\|w_{h}-\nabla u_{h}\right\|_{L^{2}(Y)}^{2} \\
&=\int_{Y} F_{\gamma}\left[\left(w_{h}, u_{h}\right)\right]\left(F_{\gamma}[(w, u)]-F_{\gamma}\left[\left(w_{h}, u_{h}\right)\right]-L_{\lambda}\left(w-w_{h}, u-u_{h}\right)\right) \\
& \quad+\left\|F_{\gamma}\left[\left(w_{h}, u_{h}\right)\right]\right\|_{L^{2}(Y)}^{2} \\
& \geq\left\|F_{\gamma}\left[\left(w_{h}, u_{h}\right)\right]\right\|_{L^{2}(Y)}^{2}-\sqrt{1-\delta}\left\|F_{\gamma}\left[\left(w_{h}, u_{h}\right)\right]\right\|_{L^{2}(Y)}\left\|\left(w-w_{h}, u-u_{h}\right)\right\|_{\lambda} \\
& \geq \frac{1}{2}\left\|F_{\gamma}\left[\left(w_{h}, u_{h}\right)\right]\right\|_{L^{2}(Y)}^{2}-\frac{1-\delta}{2}\left\|\left(w-w_{h}, u-u_{h}\right)\right\|_{\lambda}^{2},
\end{aligned}
$$

which yields the efficiency estimate upon rearranging.

\subsection{Proofs for section 3 .}

Proof of Theorem 3.9. Using the definition of the $\|\cdot\|_{\lambda_{\sigma}}$ norm and interpolation inequalities, denoting the interpolation operators on the finite element spaces by $\mathcal{I}_{h}^{\mathcal{S}^{q}}, \mathcal{I}_{h}^{\mathcal{S}^{l}}$, we find that

$$
\begin{aligned}
\inf _{\left(w_{h}^{\prime}, u_{h}^{\prime}\right) \in X_{h}} \| & \left(\nabla v^{\sigma}-w_{h}^{\prime}, v^{\sigma}-u_{h}^{\prime}\right) \|_{\lambda_{\sigma}} \\
\leq & \left\|\left(\nabla v^{\sigma}-\left(\mathcal{I}_{h}^{\mathcal{S}^{q}}\left(\nabla v^{\sigma}\right)-\int_{Y} \mathcal{I}_{h}^{\mathcal{S}^{q}}\left(\nabla v^{\sigma}\right)\right), v^{\sigma}-\mathcal{I}_{h}^{\left.\mathcal{S}^{l}\left(v^{\sigma}\right)\right)}\right)\right\|_{\lambda_{\sigma}} \\
= & \left(\left\|D\left(\nabla v^{\sigma}-\mathcal{I}_{h}^{\mathcal{S}^{q}}\left(\nabla v^{\sigma}\right)\right)\right\|_{L^{2}(Y)}^{2}+2 \lambda_{\sigma} \mid v^{\sigma}-\mathcal{I}_{h}^{\left.\mathcal{S}^{l}\left(v^{\sigma}\right)\right|_{H^{1}(Y)} ^{2}}\right. \\
& \left.\quad+\lambda_{\sigma}^{2}\left\|v^{\sigma}-\mathcal{I}_{h}^{\mathcal{S}^{l}}\left(v^{\sigma}\right)\right\|_{L^{2}(Y)}^{2}\right)^{\frac{1}{2}} \\
\leq & C_{i}\left(h^{2 \min \{r, q\}}+2 \lambda_{\sigma} h^{2 \min \{1+r, l\}}+\lambda_{\sigma}^{2} h^{2 \min \{2+r, l\}}\right)^{\frac{1}{2}}\left\|\nabla v^{\sigma}\right\|_{H^{1+r}(Y)} \\
\leq & C_{i}\left(1+2 \lambda_{\sigma}+\lambda_{\sigma}^{2}\right)^{\frac{1}{2}} h^{\min \{r, q, l\}}\left\|\nabla v^{\sigma}\right\|_{H^{1+r}(Y)}
\end{aligned}
$$

for $h>0$ sufficiently small, where $C_{i}>0$ is the constant arising in applying the interpolation inequalities. The claimed result now follows from (3.8), i.e.,

$$
\begin{aligned}
\left\|\left(\nabla v^{\sigma}-w_{h}^{\sigma}, v^{\sigma}-v_{h}^{\sigma}\right)\right\|_{\lambda_{\sigma}} & \leq C_{e}\left(\delta, \lambda_{\sigma}, n\right) \inf _{\left(w_{h}^{\prime}, u_{h}^{\prime}\right) \in X_{h}}\left\|\left(\nabla v^{\sigma}-w_{h}^{\prime}, v^{\sigma}-u_{h}^{\prime}\right)\right\|_{\lambda_{\sigma}} \\
& \leq C_{e}\left(\delta, \lambda_{\sigma}, n\right) C_{i}\left(1+\lambda_{\sigma}\right) h^{\min \{r, q, l\}}\left\|\nabla v^{\sigma}\right\|_{H^{1+r}(Y)} \\
& \leq C_{e}(\delta, \lambda, n) C_{i}(1+\lambda) h^{\min \{r, q, l\}}\left\|\nabla v^{\sigma}\right\|_{H^{1+r}(Y)}
\end{aligned}
$$

where we used $\lambda_{\sigma} \leq \lambda$ and Remark 2.8 . 
Proof of Theorem 3.11. We use Hölder and triangle inequalities, Lemma 3.7, and the error bound (3.9) to obtain

$$
\begin{aligned}
\left|\int_{Y}\left(-\sigma v_{h}^{\sigma}(\cdot ; s, p, R)\right)-H(s, p, R)\right| & \left|\int_{Y}\left(-\sigma v_{h}^{\sigma}(\cdot ; s, p, R)-H(s, p, R)\right)\right| \\
\leq & \left\|\sigma v_{h}^{\sigma}(\cdot ; s, p, R)-H(s, p, R)\right\|_{L^{2}(Y)} \\
& \quad+\sigma v_{h}^{\sigma}(\cdot ; s, p, R)-v^{\sigma}(\cdot ; s, p, R) \|_{L^{2}(Y)} \\
& \quad+\sigma(1+|p|+|R|) \\
& \lesssim\left(h^{\min \{r, q, l\}}+\sigma\right)(1+|p|+|R|) .
\end{aligned}
$$

The second part of the claim can be shown analogously.

6. Conclusion. In this work we introduced a scheme for the numerical homogenization of the fully nonlinear second-order HJB equation with Cordes coefficients, based on a novel mixed finite element method for the periodic corrector problems.

The focus of the first part of the paper was the construction and the rigorous analysis of mixed finite element approximations to the periodic solution of the HJB equation. We derived a mixed formulation for the problem and proved well-posedness as well as a priori and a posteriori error bounds. Explicit formulas for the error constants were provided, showing the asymptotic behavior of the constants in the Cordes parameters.

In the second part of the paper we focused on the numerical homogenization of HJB equations with locally periodic coefficients. Theoretical homogenization results were provided and used in the analysis of the numerical homogenization scheme. We presented and rigorously analyzed a method for the approximation of the effective Hamiltonian based on mixed finite element approximations of the periodic cell problem for the approximate corrector from the first part.

Finally, we presented numerical experiments illustrating the theoretical results. The experiments demonstrated the approximation of the effective Hamiltonian at a point as well as the approximation of the solution to the homogenized problem.

Future work will focus on the numerical homogenization of other fully nonlinear partial differential equations such as the Isaacs equation. The strong $H^{2}$ solution of Isaacs equations with Cordes coefficients has recently been discussed in [27] and can be used as a framework to study its numerical homogenization.

Acknowledgment. The second author gratefully acknowledges helpful conversations with Professor Yves Capdeboscq (Université de Paris) during the preparation of this work.

\section{REFERENCES}

[1] Y. Achdou, F. Camilli, and I. Capuzzo Dolcetta, Homogenization of Hamilton-Jacobi equations: Numerical methods, Math. Models Methods Appl. Sci., 18 (2008), pp. 11151143.

[2] O. Alvarez ANd M. BARd, Viscosity solutions methods for singular perturbations in deterministic and stochastic control, SIAM J. Control Optim., 40 (2001), pp. 1159-1188.

[3] O. Alvarez AND M. BARDI, Ergodicity, stabilization, and singular perturbations for BellmanIsaacs equations, Mem. Amer. Math. Soc., 204 (2010).

[4] M. Bebendorf, A note on the Poincaré inequality for convex domains, Z. Anal. Anwend., 22 (2003), pp. 751-756.

[5] D. Boffi, F. Brezzi, And M. Fortin, Mixed Finite Element Methods and Applications, Springer Ser. Comput. Math. 44, Springer, Berlin, 2013. 
[6] L. Caffarelli, M. G. Crandall, M. Kocan, and A. Swiech, On viscosity solutions of fully nonlinear equations with measurable ingredients, Comm. Pure Appl. Math., 49 (1996), pp. 365-397.

[7] L. A. Caffarelli, P. E. Souganidis, and L. Wang, Homogenization of fully nonlinear, uniformly elliptic and parabolic partial differential equations in stationary ergodic media, Comm. Pure Appl. Math., 58 (2005), pp. 319-361.

[8] F. Camilli and M. Falcone, An approximation scheme for the optimal control of diffusion processes, RAIRO Modél. Math. Anal. Numér., 29 (1995), pp. 97-122.

[9] F. Camilli and E. R. Jakobsen, A finite element like scheme for integro-partial differential Hamilton-Jacobi-Bellman equations, SIAM J. Numer. Anal., 47 (2009), pp. 2407-2431.

[10] F. CAMILli AND C. MARChi, Rates of convergence in periodic homogenization of fully nonlinear uniformly elliptic PDEs, Nonlinearity, 22 (2009), pp. 1481-1498.

[11] Y. CapdeboscQ, T. Sprekeler, ANd E. Süli, Finite element approximation of elliptic homogenization problems in nondivergence-form, ESAIM Math. Model. Numer. Anal., 54 (2020), pp. 1221-1257.

[12] M. Costabel and M. Dauge, Maxwell and Lamé eigenvalues on polyhedra, Math. Methods Appl. Sci., 22 (1999), pp. 243-258.

[13] M. G. Crandall, H. Ishit, And P.-L. Lions, User's guide to viscosity solutions of second order partial differential equations, Bull. Amer. Math. Soc. (N.S.), 27 (1992), pp. 1-67.

[14] L. C. Evans, The perturbed test function method for viscosity solutions of nonlinear PDE, Proc. Roy. Soc. Edinburgh Sect. A, 111 (1989), pp. 359-375.

[15] L. C. Evans, Periodic homogenisation of certain fully nonlinear partial differential equations, Proc. Roy. Soc. Edinburgh Sect. A, 120 (1992), pp. 245-265.

[16] M. Falcone ANd M. Rorro, On a variational approximation of the effective Hamiltonian, in Numerical Mathematics and Advanced Applications, Springer, Berlin, 2008, pp. 719-726.

[17] X. Feng, R. Glowinski, and M. Neilan, Recent developments in numerical methods for fully nonlinear second order partial differential equations, SIAM Rev., 55 (2013), pp. 205-267.

[18] C. Finlay And A. M. Oberman, Approximate homogenization of convex nonlinear elliptic PDEs, Commun. Math. Sci., 16 (2018), pp. 1895-1906.

[19] C. Finlay and A. M. Oberman, Approximate homogenization of fully nonlinear elliptic PDEs: Estimates and numerical results for Pucci type equations, J. Sci. Comput., 77 (2018), pp. 936-949.

[20] W. H. Fleming and H. M. Soner, Controlled Markov Processes and Viscosity Solutions, 2nd ed., Stoch. Model. Appl. Probab. 25, Springer, Berlin, 2006.

[21] D. Gallistl And E. SüLI, Mixed finite element approximation of the Hamilton-Jacobi-Bellman equation with Cordes coefficients, SIAM J. Numer. Anal., 57 (2019), pp. 592-614.

[22] R. GLOWInski, S. LeUng, AND J. QIAN, A simple explicit operator-splitting method for effective Hamiltonians, SIAM J. Sci. Comput., 40 (2018), pp. A484-A503.

[23] D. A. Gomes And A. M. OBerman, Computing the effective Hamiltonian using a variational approach, SIAM J. Control Optim., 43 (2004), pp. 792-812.

[24] H. IsHII, On uniqueness and existence of viscosity solutions of fully nonlinear second-order elliptic PDEs, Comm. Pure Appl. Math., 42 (1989), pp. 15-45.

[25] M. JEnsen, $L^{2}\left(H_{\gamma}^{1}\right)$ finite element convergence for degenerate isotropic Hamilton-JacobiBellman equations, IMA J. Numer. Anal., 37 (2017), pp. 1300-1316.

[26] M. Jensen And I. Smears, On the convergence of finite element methods for Hamilton-JacobiBellman equations, SIAM J. Numer. Anal., 51 (2013), pp. 137-162.

[27] E. L. KAWECKI AND I. SMEARS, Unified analysis of discontinuous Galerkin and $C^{0}$-interior penalty finite element methods for Hamilton-Jacobi-Bellman and Isaacs equations, ESAIM Math. Model. Numer. Anal., 55 (2021), pp. 449-478.

[28] P.-L. Lions, Optimal control of diffusion processes and Hamilton-Jacobi-Bellman equations. II. Viscosity solutions and uniqueness, Comm. Partial Differential Equations, 8 (1983), pp. 1229-1276.

[29] P.-L. Lions, A remark on Bony maximum principle, Proc. Amer. Math. Soc., 88 (1983), pp. 503-508.

[30] S. Luo, Y. Yu, and H. Zhao, A new approximation for effective Hamiltonians for homogenization of a class of Hamilton-Jacobi equations, Multiscale Model. Simul., 9 (2011), pp. 711-734.

[31] M. Neilan, A. J. Salgado, and W. Zhang, Numerical analysis of strongly nonlinear PDEs, Acta Numer., 26 (2017), pp. 137-303.

[32] A. M. Oberman, R. Takei, and A. Vladimirsky, Homogenization of metric Hamilton-Jacobi equations, Multiscale Model. Simul., 8 (2009), pp. 269-295. 
[33] J. QIAn, Two Approximations for Effective Hamiltonians Arising from Homogenization of Hamilton-Jacobi Equations, UCLA CAM report 03-39, 2003.

[34] J. Qian, H. V. Tran, AND Y. YU, Min-max formulas and other properties of certain classes of nonconvex effective Hamiltonians, Math. Ann., 372 (2018), pp. 91-123.

[35] M. V. SAfonov, Classical solution of second-order nonlinear elliptic equations, Izv. Akad. Nauk SSSR Ser. Mat., 52 (1988), pp. 1272-1287, 1328.

[36] I. Smears AND E. SÜli, Discontinuous Galerkin finite element approximation of nondivergence form elliptic equations with Cordès coefficients, SIAM J. Numer. Anal., 51 (2013), pp. 2088-2106.

[37] I. Smears AND E. SüLI, Discontinuous Galerkin finite element approximation of HamiltonJacobi-Bellman equations with Cordes coefficients, SIAM J. Numer. Anal., 52 (2014), pp. 993-1016.

[38] I. Smears And E. Süli, Discontinuous Galerkin finite element methods for time-dependent Hamilton-Jacobi-Bellman equations with Cordes coefficients, Numer. Math., 133 (2016), pp. 141-176.

[39] T. Sprekeler and H. V. Tran, Optimal Convergence Rates for Elliptic Homogenization Problems in Nondivergence-Form: Analysis and Numerical Illustrations, https://arxiv. org/abs/2009.11259.

[40] E. SüLI, A Brief Excursion into the Mathematical Theory of Mixed Finite Element Methods, Lecture Notes University of Oxford, 2013. 